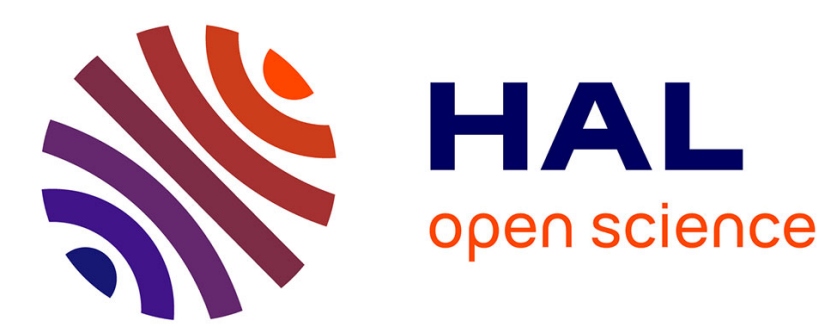

\title{
Classical and Advanced Models for Laminated Plates with Piezoelectric Layers Actuated in Shear Mode \\ M. d'Ottavio, B. Kröplin
}

\section{To cite this version:}

M. d'Ottavio, B. Kröplin. Classical and Advanced Models for Laminated Plates with Piezoelectric Layers Actuated in Shear Mode. Mechanics of Advanced Materials and Structures, 2008, 15 (3-4), pp.167 - 181. hal-01367079

\section{HAL Id: hal-01367079 \\ https://hal.science/hal-01367079}

Submitted on 10 Jan 2019

HAL is a multi-disciplinary open access archive for the deposit and dissemination of scientific research documents, whether they are published or not. The documents may come from teaching and research institutions in France or abroad, or from public or private research centers.
L'archive ouverte pluridisciplinaire HAL, est destinée au dépôt et à la diffusion de documents scientifiques de niveau recherche, publiés ou non, émanant des établissements d'enseignement et de recherche français ou étrangers, des laboratoires publics ou privés. 


\title{
Classical and Advanced Models for Laminated Plates with Piezoelectric Layers Actuated in Shear Mode
}

\author{
M. D'Ottavio, T. Wallmersperger, and B. Kröplin \\ Institute of Statics and Dynamics of Aerospace Structures, Universität Stuttgart, Stuttgart, \\ Germany
}

We discuss the two-dimensional modelling of laminated plates with embedded piezoelectric layers actuated in shear mode. Refined approaches are necessary to capture all relevant phenomena characterizing the electro-mechanical interactions. The framework of our approach is a previously established Unified Formulation, which permits the use of Equivalent Single Layer as well as Layer-Wise descriptions in conjunction with higher-order thickness approximations. Two distinct model types are proposed: classical models based on Hamilton's principle and advanced models based on a novel partially mixed four-field formulation. Advanced models are capable of $a$ priori fulfilling the interlaminar continuity of all transverse fluxes. In this paper, we adopt an analytical Navier-type closed-form solution method to solve the resulting system of coupled partial differential equations governing the static and the free-vibration problems. Different interlaminar conditions and electrode configurations are addressed. The proposed 2D models recover accurately the global and local response of exact 3D solutions available in literature. An assessment of various 2D models confirms the superiority of the advanced models with respect to classical ones. By virtue of their accuracy, the analytical solutions obtained from our higher-order advanced models could be employed for providing reference solutions for more general numerical approximation schemes like the FEM which are more suitable for practically relevant applications.

Keywords multilayered structures, piezoelectric materials, shear actuation, closed-form solution, higher-order formulations, unified formulation

\section{INTRODUCTION}

Smart structures have gained increasing attention in the past 20 years due to their capability to extend the structural response beyond the purely passive load-carrying. To this aim, composite laminated panels, i.e., plates and shells made of stacked plies or sandwich structures, have been considered for hosting layers of active materials. Piezoelectrics are perhaps the most popular active materials due to their applicability as sen- sors and actuators in the most useful engineering applications [1].

In view of a practical implementation of the attractive smart structures technology, one key step is the development of numerical models useful in structural design. Due to the usually thin panel geometries, two-dimensional (2D) structural formulations provide computationally convenient models. Piezoelectric laminates demand a consistent representation of the electro-mechanical variables and their interactions [2, 3]. Several different modelling approaches have been reviewed in $[4,5]$. These works point out the importance of a true multifield approach for an accurate local response analysis. Since the electric loadings (i.e., prescribed electric potential and/or electric charge) are possibly applied at the layer level, a smeared representation of the electric variables over the laminate crosssection proves inadequate. For this reason, hybrid approaches have been proposed which combine an Equivalent Single Layer (ESL) description for the displacement field with a Layer-Wise (LW) description for the electric potential as in, e.g., [6-8]. However, the use of ESL kinematics entailing a continuous distribution of the transverse strains in the laminate cross-section violates the physical conditions characterising the response at the layers' interfaces $[9,10]$. Within the classical "generalized displacements"-based formulations, full LW assumptions have been thus proposed for the displacement field and the electric potential as in, e.g., $[11,12]$. However, these models cannot ensure the interlaminar equilibrium due to the different electromechanical properties of adjacent layers.

Previous studies on purely elastic laminates have shown the necessity of adequately satisfying the interlaminar conditions and the role of the transverse normal stress in the fulfilment of the local equilibrium $[13,14]$. A natural tool to derive 2D models for multilayered panels satisfying a priori the interlaminar equilibrium conditions was proposed by Reissner on the basis of a partially mixed variational equation $[15,16]$. Early successful applications of the so-called Reissner's Mixed Variational Theorem (RMVT) to purely elastic laminates are reported in $[17,18]$. More recently, Carrera and coworkers [1921] compared classical (i.e., displacement-based) and advanced (i.e., RMVT-based) formulations in the framework of a Unified Formulation, which has been detailed in [22]. The Unified 
Formulation allows easy implemention of a large number of hierarchical 2D models by utilizing an extensive index notation and has been lately implemented into a commercial FEM tool [23]. Both ESL and LW descriptions can be employed for the involved variables, for which constant, linear or higher-order assumptions can be easily introduced. Since the full 3D constitutive law is retained, transverse normal effects are automatically included. The importance of a consistent submodelling technique based on a sound hierarchical approach like the Unified Formulation has been early recognized for elastic laminates [24] and recently highlighted for piezoelectric structures [25].

The Unified Formulation has been extended to piezoelectric laminates for classical generalized displacements-based models in [26-28]. Current developments aim at extending the partially mixed approach of RMVT to coupled multifield problems, in particular thermo- and electro-mechanics [29]. A partial extension of RMVT to piezoelectricity, in which the transverse stresses are a priori continuous at the interlaminar boundaries, has been presented within the Unified Formulation in [30] and successively applied in $[31,32]$. The underlying three-field formulation has been previously employed to derive a bimaterial interface and a free surface element which exactly fulfil the mechanical equilibrium [33]. A complete extension of RMVT to piezoelectricity, in which the interlaminar continuity of both the transverse stress and the transverse electric displacement is $a$ priori fulfilled, has been formally derived in [34]. On the basis of the derivation in [30], the full piezoelectric RMVT has been employed in conjunction with the Unified Formulation in $[35,36]$.

All above mentioned models of piezoelectric laminates involve the extension actuation mode, which exploits the 31effect of a piezoceramic polarized in thickness direction. In order to maximize the actuation efficiency, the piezo-layers working in the extension mode should be placed at the outer positions of the laminate. This configuration exposes the active layers to environmental loadings and impact. Moreover, the high bending strains may prove detrimental for the brittle piezoceramic materials. The implementation of piezoelectric layers into the laminate to form an adaptive sandwich structure has been thus proposed by exploiting the shear mode actuation based on the 15-effect [37]. Preliminary studies have indicated promising features of the piezoelectric sandwich structures, in particular for vibration damping applications [38].

Many proposed models for piezoelectric sandwich beams or plates rely on classical Kirchhoff assumptions for the exterior faces [38-41] and apply the first-order [38-40] or the third-order shear deformation theory [41] for the piezoelectric core. Analytical solutions for continuous piezoelectric sandwich structures have been obtained by the state space approach for simple shear-deformable beam formulations with equivalent single layer kinematics in [42]. Exact solutions based on the three-dimensional coupled piezoelectric formulation are given in [43] for the static problem in a generalized plane state of deformation (cylindrical bending). Exact 3D solutions for the vi- bration problem in cylindrical bending are reported along with an active damping analysis for sandwich plates [44] and cylindrical shells [45]. While the works cited above have the generalized displacements as independent unknowns, a mixed formulation involving the transverse fluxes (i.e., the transverse stresses and the transverse electric displacement) as additional independent variables is used in $[46,47]$. The mixed formulation is employed in conjunction with the state space approach to obtain exact 3D solutions for the static (sensor and actuator) and freevibration response of sandwich structures with a shear actuated piezoelectric core.

In this work, the Unified Formulation is applied to laminates with embedded, shear actuated piezoelectric layers. Both classical (i.e., generalized displacements-based) and advanced (i.e., based on the piezoelectric extension of RMVT) modelling approaches are presented and compared. Particular attention is devoted to the interlaminar conditions and the electric boundary conditions. Numerical results are obtained for different configurations within a closed-form solution based on Navier's method for static and free-vibration problems. An outline of this article is as follows: Section 2 recalls the 3D governing equations and introduces the employed notation. In Section 3, the classical and advanced 2D models are derived on the basis of two variational equations (given in Sections 3.1 and 3.2, respectively) and according to the Unified Formulation (Section 3.3). The closed-form solution is discussed along with the interlaminar conditions in Section 4. The validation of the proposed formulation for both the static problem and the free-vibration problem, is performed in Section 5 by comparing the results of the most accurate 2D models with exact 3D solutions available in literature. The capability of the Unified Formulation is demonstrated by means of a numerical assessment of a free-vibration problem in Section 6. Finally, in Section 7 conclusions are given and an outlook is suggested.

\section{CONTINUUM MECHANICS OF PIEZOELECTRIC LAMINATED PLATES}

We consider a laminated plate consisting of $N_{l}$ homogeneous layers, which is described in terms of the in-plane coordinates $x, y$ (with $\Omega=[x \times y]$ ) and the thickness coordinate $z$ (Figure 1). Within the small signal range and considering only small displacements, the linearized gradient relations defining

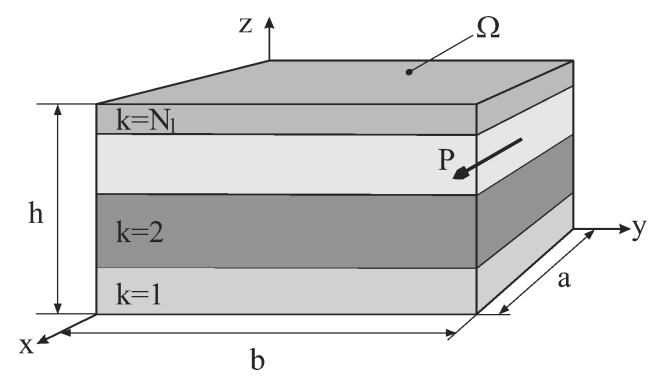

FIG. 1. Geometry description and employed notation for a multilayered plate. $P$ indicates the direction of polarisation of piezoelectric layers. 
the strain and the electric fields $\epsilon, \mathbf{E}$ are employed for each layer $k$ [48]:

$$
\begin{aligned}
\mathbf{\epsilon}_{p}^{k} & =\left[\epsilon_{1}^{k}, \epsilon_{2}^{k}, \epsilon_{6}^{k}\right]^{T}=\mathbf{B}_{p} \mathbf{u}^{k} ; \\
\mathbf{\epsilon}_{n}^{k} & =\left[\epsilon_{4}^{k}, \epsilon_{5}^{k}, \epsilon_{3}^{k}\right]^{T}=\mathbf{B}_{n \Omega} \mathbf{u}^{k}+\mathbf{B}_{n z} \mathbf{u}^{k} ; \\
\mathbf{E}_{p}^{k} & =\left[\mathrm{E}_{x}^{k}, \mathrm{E}_{y}^{k}\right]^{T}=\mathbf{B}_{e \Omega} \Phi^{k} ; \\
\mathbf{E}_{n}^{k} & =\mathrm{E}_{z}^{k}
\end{aligned}
$$

where $\mathbf{u}^{k}$ and $\Phi^{k}$ are the displacement vector and the electric potential in the $k^{\text {th }}$ layer and the superscript $T$ denotes transposition. The differential arrays $\mathbf{B}$ contain the partial derivative operators with respect to the spatial coordinates $x_{i}, \frac{\partial}{\partial x_{i}}=\partial_{x_{i}}$, and read

$$
\begin{aligned}
\mathbf{B}_{p} & =\left[\begin{array}{ccc}
\partial_{x} & 0 & 0 \\
0 & \partial_{y} & 0 \\
\partial_{y} & \partial_{x} & 0
\end{array}\right] ; \quad \mathbf{B}_{n \Omega}=\left[\begin{array}{ccc}
0 & 0 & \partial_{x} \\
0 & 0 & \partial_{y} \\
0 & 0 & 0
\end{array}\right] ; \quad \mathbf{B}_{e \Omega}=\left[\begin{array}{l}
-\partial_{x} \\
-\partial_{y}
\end{array}\right] \\
\mathbf{B}_{n z} & =\left[\begin{array}{ccc}
\partial_{z} & 0 & 0 \\
0 & \partial_{z} & 0 \\
0 & 0 & \partial_{z}
\end{array}\right] ; \quad \mathbf{B}_{e z}=-\partial_{z}
\end{aligned}
$$

Note that the standard contracted Voigt-Kelvin notation is employed. The in-plane (subscript $p$ ) and transverse (subscript $n$ ) contributions have been split in view of the subsequent 2D modelling technique.

The divergence equations stating the mechanical and electrical equilibrium for each layer $k$ are written in the general dynamic case as

$$
\begin{aligned}
\nabla \boldsymbol{\sigma}^{k}+\mathbf{b}^{k} & =\rho^{k} \ddot{\mathbf{u}} ; \\
\nabla \mathbf{D}^{k} & =0 .
\end{aligned}
$$

A dot stays for temporal derivative and double dots indicate acceleration. $\rho$ denotes the mass density and $\mathbf{b}$ the vector of body forces. Since the electromagnetic wavelengths are much longer than the elastic ones of the same frequency, we make use of the hypothesis of a quasi-static electric field [49].

Conditions for the mechanical and electrical fluxes as well as for the generalized displacements are prescribed on the boundary $\Gamma$ of the multilayered domain according to

$$
\begin{aligned}
& \mathbf{t}=\boldsymbol{\sigma} \cdot \mathbf{n}_{t}=\overline{\mathbf{t}} \quad \text { on } \Gamma_{t} ; \quad \mathbf{q}=-\mathbf{D} \cdot \mathbf{n}_{q}=\bar{q} \quad \text { on } \Gamma_{q} ; \\
& \mathbf{u}=\overline{\mathbf{u}} \quad \text { on } \Gamma_{u} \text {; } \\
& \Phi=\bar{\Phi} \quad \text { on } \Gamma_{\Phi}
\end{aligned}
$$

For simplicity, the common assumption $\Gamma=\Gamma_{t} \cup \Gamma_{u}=$ $\Gamma_{q} \cup \Gamma_{\Phi}$ has been introduced. $\mathbf{n}$ is the outward normal to the boundary portion $\Gamma, \mathbf{t}$ represents the traction component, $q$ denotes the surface charge density and a bar indicates prescribed values.

The material behavior is expressed within the linear piezoelectricity theory according to [50]. Splitting the stress $\sigma$ and electric displacement $\mathbf{D}$ tensors in their in-plane and transverse components according to Eq. (1) the inverse and direct effects are expressed as

$$
\begin{aligned}
\boldsymbol{\sigma}_{p} & =\mathbf{C}_{p p}^{e} \boldsymbol{\epsilon}_{p}+\mathbf{C}_{p n}^{e} \boldsymbol{\epsilon}_{n}-\mathbf{e}_{p p}^{T} \mathbf{E}_{p}-\mathbf{e}_{n p}^{T} \mathbf{E}_{n} ; \\
\boldsymbol{\sigma}_{n} & =\mathbf{C}_{p n}^{e T} \boldsymbol{\epsilon}_{p}+\mathbf{C}_{n n}^{e} \boldsymbol{\epsilon}_{n}-\mathbf{e}_{p n}^{T} \mathbf{E}_{p}-\mathbf{e}_{n n}^{T} \mathbf{E}_{n} ; \\
\mathbf{D}_{p} & =\mathbf{e}_{p p} \boldsymbol{\epsilon}_{p}+\mathbf{e}_{p n} \boldsymbol{\epsilon}_{n}+\varepsilon_{p p}^{s} \mathbf{E}_{p}+\varepsilon_{p n}^{s} \mathbf{E}_{n} \\
\mathbf{D}_{n} & =\mathbf{e}_{n p} \boldsymbol{\epsilon}_{p}+\mathbf{e}_{n n} \boldsymbol{\epsilon}_{n}+\varepsilon_{p n}^{s T} \mathbf{E}_{p}+\varepsilon_{n n}^{s} \mathbf{E}_{n}
\end{aligned}
$$

where the superscript $k$ has been dropped out for clarity, and the superscripts $e$ and $s$ indicate that the material coefficients are taken at constant electric field and at constant strain, respectively. A purely mechanical behavior is obtained by setting the piezoelectric coefficients $e$ to zero. By ordering the components of the fluxes according to the scheme given in Eq. (1) i.e., $\boldsymbol{\sigma}_{p}=\left[\sigma_{1} \sigma_{2} \sigma_{6}\right], \boldsymbol{\sigma}_{n}=\left[\sigma_{4} \sigma_{5} \sigma_{3}\right], \mathbf{D}_{p}=\left[\mathrm{D}_{x} \mathrm{D}_{y}\right]$ and $\mathbf{D}_{n}=\mathrm{D}_{z}$ - the tensors of elastic, dielectric and piezoelectric coefficients for an in-plane ( $x$ axis) polarized material read [34]

$$
\begin{aligned}
& \mathbf{C}_{p p}^{e}= {\left[\begin{array}{ccc}
C_{11}^{e} & C_{12}^{e} & 0 \\
C_{12}^{e} & C_{22}^{e} & 0 \\
0 & 0 & C_{66}^{e}
\end{array}\right] ; } \\
& \mathbf{C}_{p n}^{e}=\left[\begin{array}{ccc}
0 & 0 & C_{13}^{e} \\
0 & 0 & C_{23}^{e} \\
0 & 0 & 0
\end{array}\right] ; \quad \mathbf{C}_{n n}^{e}=\left[\begin{array}{ccc}
C_{44}^{e} & 0 & 0 \\
0 & C_{55}^{e} & 0 \\
0 & 0 & C_{33}^{e}
\end{array}\right] ; \\
& \boldsymbol{\varepsilon}_{p p}^{s}=\left[\begin{array}{cc}
\varepsilon_{11}^{s} & 0 \\
0 & \varepsilon_{22}^{s}
\end{array}\right] ; \quad \boldsymbol{\varepsilon}_{p n}^{s}=\left[\begin{array}{l}
0 \\
0
\end{array}\right] ; \boldsymbol{\varepsilon}_{n n}^{s}=\varepsilon_{33}^{s} ; \\
& \mathbf{e}_{p p}= {\left[\begin{array}{ccc}
e_{11} & e_{12} & 0 \\
0 & 0 & e_{26}
\end{array}\right] ; \mathbf{e}_{p n}=\left[\begin{array}{lll}
0 & 0 & e_{13} \\
0 & 0 & 0
\end{array}\right] ; \mathbf{e}_{n p}=\left[\begin{array}{lll}
0 & 0 & 0
\end{array}\right] ; } \\
& \mathbf{e}_{n n}= {\left[\begin{array}{lll}
0 & e_{35} & 0
\end{array}\right] . }
\end{aligned}
$$

The above relations have been written in the coordinate system defined by the principal directions of an orthotropic material. By building the stiffness arrays, the rotation angle $\theta^{k}$ defining the relative orientation between the material axes and the structural reference frame must be taken into account (the required transformations can be found in, e.g., [51]). Throughout this work, the polarization axis of piezoelectric layers is assumed to coincide with the structural $x$ axis. In the constitutive behavior of elastic layers the piezoelectric coefficients are set to zero.

\subsection{Interlaminar conditions}

All layers in the laminate are assumed to be perfectly bonded. Therefore, the displacement and the transverse stress fields are interlaminar continuous:

$$
\mathbf{u}^{k t}-\mathbf{u}^{(k+1) b}=\mathbf{0} ; \quad \boldsymbol{\sigma}_{n}^{k t}-\boldsymbol{\sigma}_{n}^{(k+1) b}=\mathbf{0},
$$

where the superscripts $k t$ and $(k+1) b$ denote the top of the $k^{\text {th }}$ layer and the bottom of the $(k+1)^{\text {th }}$ layer, respectively. As far as 
the electrical variables are concerned, we distinguish a "bare" interface from an electroded interface. Since the main attention is given to the mechanical response of the laminate, there is no need to explicitly model the thin electrodes as additional layers, and a representation of the effects induced on the throughthickness distributions of the field variables is sufficient. The following interlaminar conditions are distinguished:

1. Interface without electrodes: the electric potential and the transverse electric displacement are continuous:

$$
\Phi^{k t}-\Phi^{(k+1) b}=0 ; \quad \mathrm{D}_{z}^{k t}-\mathrm{D}_{z}^{(k+1) b}=0 .
$$

2. Electroded interface: depending on the electric conditions defined on the electrode, we distinguish the following configurations:

- Prescribed potential: if a potential $\bar{\Phi}$ is given, a discontinuity of $\mathrm{D}_{z}$ is possible because the electrode is capable of collecting a surface charge $q$ :

$$
\Phi^{k t}=\Phi^{(k+1) b}=\bar{\Phi} ; \quad \mathrm{D}_{z}^{k t}-\mathrm{D}_{z}^{(k+1) b}=q
$$

Whenever an electric potential is prescribed on an electrode, the collected charge is a free variable.

- Prescribed charge: the discontinuity of $\mathrm{D}_{z}$ is prescribed by the applied charge density $\bar{q}$ according to the second of Eq. (4a) [52]

$$
\Phi^{k t}-\Phi^{(k+1) b}=0 ; \quad \mathrm{D}_{z}^{k t}-\mathrm{D}_{z}^{(k+1) b}=\bar{q} .
$$

In the special case of an open-circuited electrode (i.e., $\bar{q}=0$ ), the prescribed jump vanishes and the interlaminar continuity conditions in Eq. (8) are recovered. Whenever an electric charge is prescribed on an electrode, the corresponding electric potential is a free variable. However, in literature the continuity of the electric potential is often relaxed in this case $[46,47]$.

\section{TWO-DIMENSIONAL MODELS: THE UNIFIED FORMULATION}

The variational procedure is employed to establish the consistent set of governing equations and boundary conditions for the piezoelectric laminate. Classical formulations are obtained from the well-established extension of Hamilton's principle to piezoelectric media [49]. Alternatively, "advanced" formulations are proposed on the basis of a novel four-field partially mixed variational equation corresponding to the extension of Reissner's Mixed Variational Theorem (RMVT) to piezoelectric laminates [34]. Within an axiomatic modelling technique, we introduce a priori assumptions for the thickness behaviour of the three-dimensional field variables. The peculiar index notation of the Unified Formulation is employed to derive a hierarchic series of models within both the classical and the advanced formulations.

\subsection{Classical Models: Hamilton's Principle}

Classical formulations utilize the generalized displacements as independent unknowns and rely on Hamilton's principle applied to piezoelectric media [49]. Following the usual procedure for laminated structures (see, e.g., [51]), we write the variational equation for each layer (the index $k$ is dropped out for clarity):

$$
\begin{aligned}
\int_{t_{0}}^{t_{1}} & {\left[\int_{\Omega_{k}} \int_{h_{k}} \delta \boldsymbol{\epsilon}_{p G}^{T} \boldsymbol{\sigma}_{p C}+\delta \epsilon_{n G}^{T} \boldsymbol{\sigma}_{n C}+\delta \mathbf{u}^{T}(\rho \ddot{\mathbf{u}}-\mathbf{b}) \mathrm{d} z \mathrm{~d} \Omega\right.} \\
& \left.-\int_{\Omega_{k}} \int_{h_{k}} \delta \mathbf{E}_{p G}^{T} \mathbf{D}_{p C}+\delta \mathbf{E}_{n G}^{T} \mathbf{D}_{n C} \mathrm{~d} z \mathrm{~d} \Omega\right] \mathrm{d} t \\
& -\int_{t_{0}}^{t_{1}}\left[\int_{\Gamma_{t}^{k}} \delta \mathbf{u}^{T} \overline{\mathbf{t}} \mathrm{d} \Gamma-\int_{\Gamma_{q}^{k}} \delta \Phi \bar{q} \mathrm{~d} \Gamma\right] \mathrm{d} t=\int_{t_{0}}^{t_{1}} \delta W_{e}^{k} \mathrm{~d} t .
\end{aligned}
$$

$\delta W_{e}^{k}$ represents the virtual work done by the external applied forces. Subscripts $G$ and $C$ indicate that the quantities are defined by the gradient relations in Eq. (1) and by the constitutive equations reported in Eq. (5), respectively.

\subsection{Advanced Models: The Piezoelectric Reissner Equation}

The derivation of the partially mixed variational statement follows the guidelines presented in [30] and yields the following expression (see also [29, 34]):

$$
\begin{aligned}
\int_{t_{0}}^{t} & {\left[\int_{\Omega_{k}} \int_{h_{k}} \delta \mathbf{\epsilon}_{p G}^{T} \boldsymbol{\sigma}_{p C}+\delta \mathbf{\epsilon}_{n G}^{T} \boldsymbol{\sigma}_{n M}+\delta \boldsymbol{\sigma}_{n M}^{T}\left(\boldsymbol{\epsilon}_{n G}-\mathbf{\epsilon}_{n C}\right)\right.} \\
& +\delta \mathbf{u}^{T}(\rho \ddot{\mathbf{u}}-\mathbf{b}) \mathrm{d} z \mathrm{~d} \Omega \\
& -\int_{\Omega_{k}} \int_{h_{k}} \delta \mathbf{E}_{p G}^{T} \mathbf{D}_{p C}+\delta \mathbf{E}_{n G}^{T} \mathbf{D}_{n M} \\
& \left.+\delta \mathbf{D}_{n M}^{T}\left(\mathbf{E}_{n G}-\mathbf{E}_{n C}\right) \mathrm{d} z \mathrm{~d} \Omega\right] \mathrm{d} t \\
& -\int_{t_{0}}^{t}\left[\int_{\Gamma_{t}^{k}} \delta \mathbf{u}^{T} \overline{\mathbf{t}} \mathrm{d} \Gamma-\int_{\Gamma_{q}^{k}} \delta \Phi \bar{q} \mathrm{~d} \Gamma\right] \mathrm{d} t=\int_{t_{0}}^{t_{1}} \delta W_{e}^{k} \mathrm{~d} t .
\end{aligned}
$$

Note that the external work definition as well as all boundary terms remain unchanged with respect to Hamilton's principle. The partially mixed equation allows to formulate an independent assumption for the transverse fluxes and, hence, to model them to exactly fulfil the interlaminar continuity (subscript $M$ ). Quantities defined by the gradient relations in Eq. (1) are denoted by the subscript $G$. A subscript $C$ indicates that the quantity is defined by the constitutive equations associated to the 
partially mixed formulation, which are expressed as follows:

$$
\begin{aligned}
\boldsymbol{\sigma}_{p C} & =\mathbf{C}_{p p}^{M} \boldsymbol{\epsilon}_{p G}+\mathbf{C}_{p n}^{M} \boldsymbol{\sigma}_{n M}-\mathbf{e}_{p p}^{M T} \mathbf{E}_{p G}-\mathbf{e}_{n p}^{M T} \mathbf{D}_{n M} \\
\mathbf{\epsilon}_{n C} & =-\mathbf{C}_{p n}^{M T} \mathbf{\epsilon}_{p G}+\mathbf{C}_{n n}^{M} \boldsymbol{\sigma}_{n M}+\mathbf{e}_{p n}^{M T} \mathbf{E}_{p G}+\mathbf{e}_{n n}^{M T} \mathbf{D}_{n M} \\
\mathbf{D}_{p C} & =\mathbf{e}_{p p}^{M} \boldsymbol{\epsilon}_{p G}+\mathbf{e}_{p n}^{M} \boldsymbol{\sigma}_{n M}+\varepsilon_{p p}^{M} \mathbf{E}_{p G}+\varepsilon_{p n}^{M} \mathbf{D}_{n M} \\
\mathbf{E}_{n C} & =-\mathbf{e}_{n p}^{M} \boldsymbol{\epsilon}_{p G}-\mathbf{e}_{n n}^{M} \boldsymbol{\sigma}_{n M}-\varepsilon_{p n}^{M T} \mathbf{E}_{p G}+\varepsilon_{n n}^{M} \mathbf{D}_{n M}
\end{aligned}
$$

The mixed coefficient arrays (superscript $M$ ) can be expressed in terms of the elasticity, dielectric and piezoelectric coefficients introduced in Eq. (6) in the following manner:

$$
\begin{aligned}
& \mathbf{C}_{p p}^{M}= {\left[\begin{array}{ccc}
C_{11}^{e}-\frac{C_{13}^{e}}{C_{33}^{e}} & C_{12}^{e}-\frac{C_{13}^{e} C_{33}^{e}}{C_{33}^{e}} & 0 \\
C_{12}^{e}-\frac{C_{13}^{e} C_{23}^{e}}{C_{33}^{2}} & C_{22}^{e}-\frac{C_{23}^{e}}{C_{33}^{e}} & 0 \\
0 & 0 & C_{66}^{e}
\end{array}\right] ; \quad \mathbf{C}_{p n}^{M}=\left[\begin{array}{ccc}
0 & 0 & \frac{C_{13}^{e}}{C_{33}^{e}} \\
0 & 0 & \frac{C_{23}^{e}}{C_{33}^{e}} \\
0 & 0 & 0
\end{array}\right] ; } \\
& \mathbf{C}_{n n}^{M}=\left[\begin{array}{ccc}
\frac{1}{C_{44}^{e}} & 0 & 0 \\
0 & \frac{1}{C_{55}^{e}}\left[\begin{array}{cc}
1-\frac{e_{35}^{2}}{\kappa C_{55}^{e}} \\
0
\end{array}\right] & 0 \\
0 & \frac{1}{C_{33}^{e}}
\end{array}\right] ; \quad \varepsilon_{p p}^{M}=\left[\begin{array}{ccc}
\varepsilon_{11}^{s}+\frac{e_{13}^{2}}{C_{33}^{e}} & 0 \\
0 & \varepsilon_{22}^{s}
\end{array}\right] ; \\
& \varepsilon_{p n}^{M}=\left[\begin{array}{l}
0 \\
0
\end{array}\right] ; \quad \varepsilon_{n n}^{M}=\frac{1}{\kappa} ; \quad \mathbf{e}_{p p}^{M}=\left[\begin{array}{ccc}
e_{11}-\frac{C_{13}^{e} e_{13}}{C_{33}^{e}} & e_{12}-\frac{C_{23}^{e} e_{13}}{C_{33}^{e}} & 0 \\
0 & 0 & e_{26}
\end{array}\right] ; \\
& \mathbf{e}_{p n}^{M}=\left[\begin{array}{ccc}
0 & 0 & \frac{e_{13}}{C_{33}^{e}} \\
0 & 0 & 0
\end{array}\right] ; \quad \mathbf{e}_{n p}^{M}=\left[\begin{array}{lll}
0 & 0 & 0
\end{array}\right] ; \\
& \mathbf{e}_{n n}^{M}=\left[\begin{array}{lll}
0 & \frac{e_{35}}{\kappa C_{55}^{e}} & 0
\end{array}\right] ; \quad \kappa=\varepsilon_{33}^{s}+\frac{e_{35}^{2}}{C_{55}^{e}} .
\end{aligned}
$$

\subsection{Thickness Assumptions}

The thickness assumptions for the generic independent variable $\mathcal{U}^{k}(x, y, z)$ and its virtual variation $\delta \mathcal{U}^{k}(x, y, z)$ are postulated as:

$$
\mathcal{U}^{k}\left(x, y, z_{k}\right)=F_{s}^{k}\left(z_{k}\right) \tilde{\mathcal{U}}_{s}^{k}(x, y) \quad \text { with } s=0,1,2, \ldots N
$$

$$
\delta \mathcal{U}^{k}\left(x, y, z_{k}\right)=F_{\tau}^{k}\left(z_{k}\right) \tilde{\mathcal{U}}_{\tau}^{k}(x, y) \quad \text { with } \tau=0,1,2, \ldots N .
$$

To accomplish a hierarchic modelling technique, the order $N$ of the polynomial employed for the postulated thickness behavior remains a free parameter. In this work, linear $(N=1)$ up to fourth-order $(N=4)$ polynomials are used for the thickness assumptions. Additionally, the level at which the thickness assumptions are introduced is a further free choice of the formulation. Two different laminate descriptions can be thus implemented, namely [51]:

- Layer-Wise (LW) descriptions are obtained by introducing the thickness assumptions for each layer separately. An appropriate assembly procedure is employed to build the multilayered contributions by taking into account the interface conditions. An
LW description possesses a number of Degrees Of Freedom (DOF) which depends on the number of layers utilized for subdividing the laminate.

- Equivalent Single Layer (ESL) descriptions are obtained by applying the thickness assumptions throughout the whole multilayered cross-section. The number of DOF is, hence, independent from the number of layers constituting the laminate. To improve the interlaminar response of ESL models by allowing a discontinuous slope at the interfaces, Murakami's Zig-Zag function can be superimposed to the standard ESL displacement field [17, 53].

The expressions for the employed polynomial functions for the ESL and LW descriptions can be found in numerous previous works based on the Unified Formulation (e.g., $[22,26,27,31,35])$. By extensively using the index notation (specifically, the expansion indexes $\tau, s$ and the layer index $k$ ), the Unified Formulation is capable of representing in a compact and implementation-friendly manner a large variety of 2D models. The models employed in this work are summarized in the ; following along with the acronyms used for their designation:

- Classical Models: The electric potential is always assumed within an LW description. The displacement field $\mathbf{u}$ can be chosen to be either ESL or LW. The acronym is constructed according to Figure 2. The polynomial order $N$ of the thickness assumptions is the same for all variables.

- Advanced Models: The approximations for the generalized displacements are introduced as in classical models (i.e., u ESL or LW, $\Phi$ always LW); the additional independent approximations for the transverse fluxes are introduced always at layer level. Again, the same order $N$ is considered for all variables. An " $\mathrm{M}$ " in the acronym indicates the use of the mixed formulation (Figure 2).

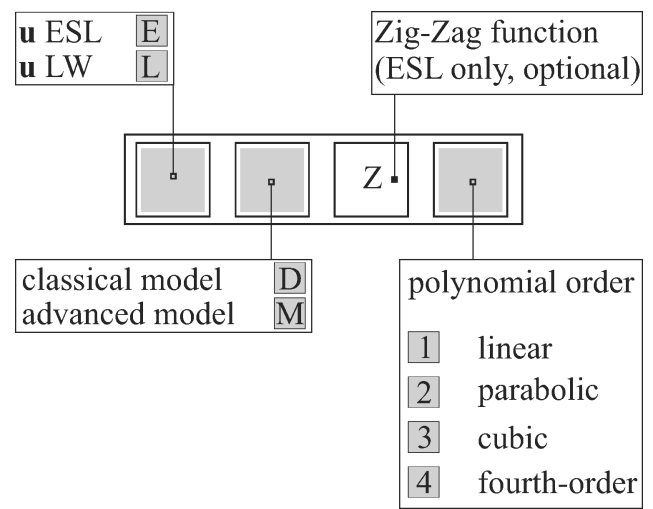

FIG. 2. Construction of the employed acronyms for the numerical analysis. 
TABLE 1

Material data employed in the numerical analyses

\begin{tabular}{|c|c|c|c|c|c|}
\hline \multicolumn{6}{|c|}{$\begin{array}{c}\text { In-plane }(x \text {-axis) polarised } \\
\text { piezoceramic PZT-5H (from }[46,47])\end{array}$} \\
\hline$C_{11}^{e}$ & 117 & [GPa] & $e_{12}=e_{13}$ & -6.5 & {$\left[\mathrm{C} / \mathrm{m}^{2}\right]$} \\
\hline$C_{22}^{e}=C_{33}^{e}$ & 126 & [GPa] & $e_{11}$ & 23.3 & {$\left[\mathrm{C} / \mathrm{m}^{2}\right]$} \\
\hline$C_{12}^{e 2}=C_{13}^{e 3}$ & 84.1 & [GPa $]$ & $e_{26}=e_{35}$ & 17 & {$\left[\mathrm{C} / \mathrm{m}^{2}\right]$} \\
\hline$C_{23}^{e}$ & 79.5 & [GPa] & $\varepsilon_{11}^{s}$ & $13010^{-10}$ & {$[\mathrm{C} / \mathrm{Vm}]$} \\
\hline$C_{55}^{e s}=C_{66}^{e}$ & 23 & [GPa] & $\varepsilon_{22}^{s}=\varepsilon_{33}^{s}$ & $150.310^{-10}$ & {$[\mathrm{C} / \mathrm{Vm}]$} \\
\hline$C_{44}^{e}$ & 23 & [GPa] & $\rho$ & 7500 & {$\left[\mathrm{~kg} / \mathrm{m}^{3}\right]$} \\
\hline \multicolumn{6}{|c|}{$0^{\circ}$ Graphite-Epoxy (from $\left.[43,47]\right)$} \\
\hline$C_{11}^{e}$ & 183.443 & [GPa] & $e_{12}=e_{13}$ & - & \\
\hline$C_{22}^{e}=C_{33}^{e}$ & 11.662 & [GPa] & $e_{11}$ & - & \\
\hline$C_{12}^{e}=C_{13}^{e}$ & 4.363 & [GPa] & $e_{26}=e_{35}$ & - & \\
\hline$C_{23}^{e}$ & 3.918 & {$[\mathrm{GPa}]$} & $\varepsilon_{11}^{s}$ & $153.010^{-10}$ & [C/Vm] \\
\hline$C_{55}^{e}=C_{66}^{e}$ & 7.170 & [GPa $]$ & $\varepsilon_{22}^{s}=\varepsilon_{33}^{s}$ & $153.010^{-10}$ & {$[\mathrm{C} / \mathrm{Vm}]$} \\
\hline$C_{44}^{e}$ & 2.870 & {$[\mathrm{GPa}]$} & $\rho$ & 1580 & {$\left[\mathrm{~kg} / \mathrm{m}^{3}\right]$} \\
\hline
\end{tabular}

\section{CLOSED-FORM SOLUTION}

The two-dimensional models are obtained following the standard procedure to eliminate the thickness coordinate. The thickness assumptions are introduced into the variational equation along with the associated subsidiary conditions (i.e., the gradient relations and the respective constitutive equations). The differentiations defined by the operators in Eq. (2b) are carried out and the thickness coordinate $z_{k}$ is finally eliminated by pre-integrating over the layer thickness $h_{k}$. In view of the subsequent analytical solution of the resulting $2 \mathrm{D}$ problem, the strong form of the governing partial differential equations is first recovered by integrating by parts the appropriate terms of the domain integrals.

Analytical solutions can be found for problems with certain lamination schemes and boundary conditions. We consider a rectangular plate of dimension $x=[0, a], y=[0, b]$ and thickness $h$ (Fig. 1) with a cross-ply lay-up which prevents the coupling between direct stresses and shearing strains (i.e., $C_{16}^{e}=C_{26}^{e}=C_{36}^{e}=0$ ) as well as the coupling between the transverse shear components (i.e., $C_{45}^{e}=0$ ). The lateral boundaries are considered to be mechanically simply supported:

$$
\begin{array}{ll}
u_{y}^{k}=u_{z}^{k}=0 & \text { at } x=0, a ; \\
u_{x}^{k}=u_{z}^{k}=0 & \text { at } y=0, b .
\end{array}
$$

Electrically, the lateral edges perpendicular to the polarization direction (i.e., the $x$-direction in the present case) are supposed to be insulated (charge-free), while the remaining edges pair is taken to be grounded (potential-free):

$$
\begin{aligned}
D_{x}^{k}=0 & \text { at } x=0, a ; \\
\Phi^{k}=0 & \text { at } y=0, b .
\end{aligned}
$$

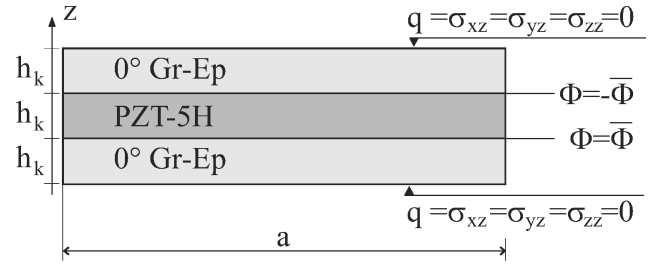

(a) Actuator configuration: electrical loading

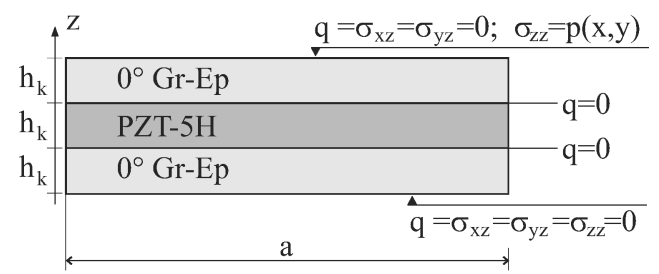

(b) Sensor configuration: mechanical loading

FIG. 3. Laminate configurations for the static and free-vibration problems.

Under the above conditions, the Navier solution technique can be employed to find solutions which exactly fulfill the governing partial differential equations and the boundary conditions. By limiting the analysis to a single term of the infinite double series expansion, a closed-form solution is obtained. The in-plane distribution of the field variables reads

$$
\begin{aligned}
& {\left[\tilde{u}_{x}^{k}(x, y) ; \tilde{\sigma}_{x z}^{k}(x, y)\right]=\left[\hat{u}_{x}^{k} ; \hat{\sigma}_{x z}^{k}\right] \cos \left(\frac{m_{x} \pi x}{a}\right) \sin \left(\frac{m_{y} \pi y}{b}\right) ;} \\
& {\left[\tilde{u}_{y}^{k}(x, y) ; \tilde{\sigma}_{y z}^{k}(x, y)\right]=\left[\hat{u}_{y}^{k} ; \hat{\sigma}_{y z}^{k}\right] \sin \left(\frac{m_{x} \pi x}{a}\right) \cos \left(\frac{m_{y} \pi y}{b}\right) ;} \\
& {\left[\tilde{u}_{z}^{k}(x, y) ; \tilde{\sigma}_{z z}^{k}(x, y)\right]=\left[\hat{u}_{z}^{k} ; \hat{\sigma}_{z z}^{k}\right] \sin \left(\frac{m_{x} \pi x}{a}\right) \sin \left(\frac{m_{y} \pi y}{b}\right) ;} \\
& {\left[\tilde{\Phi}^{k}(x, y) ; \tilde{\mathrm{D}}_{z}^{k}(x, y)\right]=\left[\hat{\Phi}^{k} ; \hat{\mathrm{D}}_{z}^{k}\right] \cos \left(\frac{m_{x} \pi x}{a}\right) \sin \left(\frac{m_{y} \pi y}{b}\right),}
\end{aligned}
$$

with $m_{x}$ and $m_{y}$ being the number of half-waves in the $x$ and $y$ direction, respectively. The single-term expressions for the external loadings are assumed as

$$
\begin{aligned}
& p(x, y)=p_{0} \sin \left(\frac{m_{x} \pi x}{a}\right) \sin \left(\frac{m_{y} \pi y}{b}\right) ; \\
& \bar{q}(x, y)=\bar{q}_{0} \cos \left(\frac{m_{x} \pi x}{a}\right) \sin \left(\frac{m_{y} \pi y}{b}\right) .
\end{aligned}
$$

Introducing the above functions into the $2 \mathrm{D}$ governing equations of the four-field partially mixed model, the following set of coupled algebraic equations is obtained:

$$
\begin{gathered}
\delta \hat{\mathbf{u}}_{\tau}^{k}: \quad \hat{\mathbf{K}}_{u u}^{k \tau s} \hat{\mathbf{u}}_{s}^{k}+\hat{\mathbf{K}}_{u v}^{k \tau s} \hat{\Phi}_{s}^{k}+\hat{\mathbf{K}}_{u s}^{k \tau s}\left(\hat{\boldsymbol{\sigma}}_{n}^{k}\right)_{s}+\hat{\mathbf{K}}_{u d}^{k \tau s}\left(\hat{\mathrm{D}}_{n}^{k}\right)_{s} \\
=\hat{\mathbf{M}}^{k \tau s} \ddot{\mathbf{u}}_{s}^{k}+\hat{\mathbf{p}}_{\tau}^{k}
\end{gathered}
$$



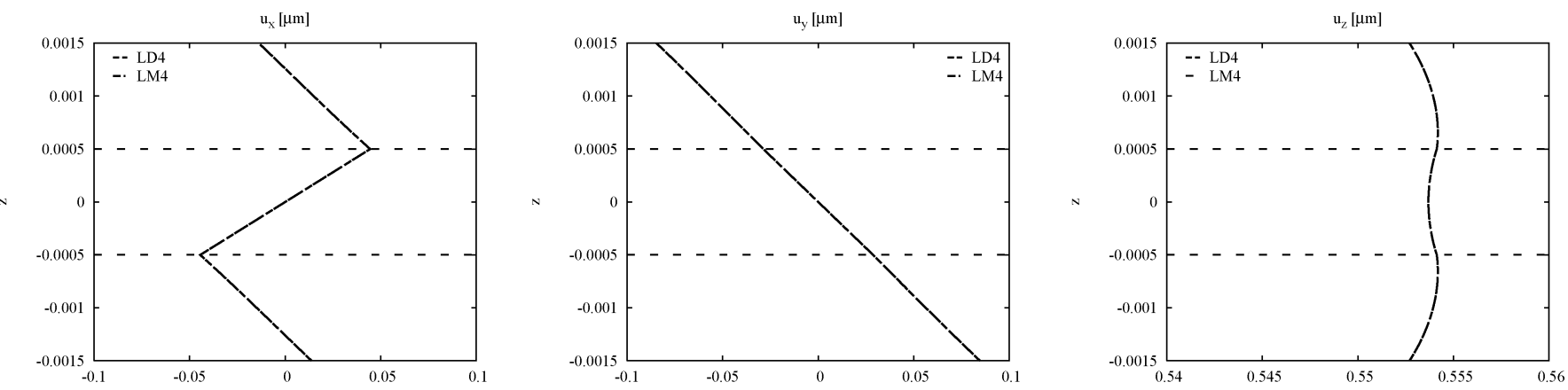

(a) Displacement components
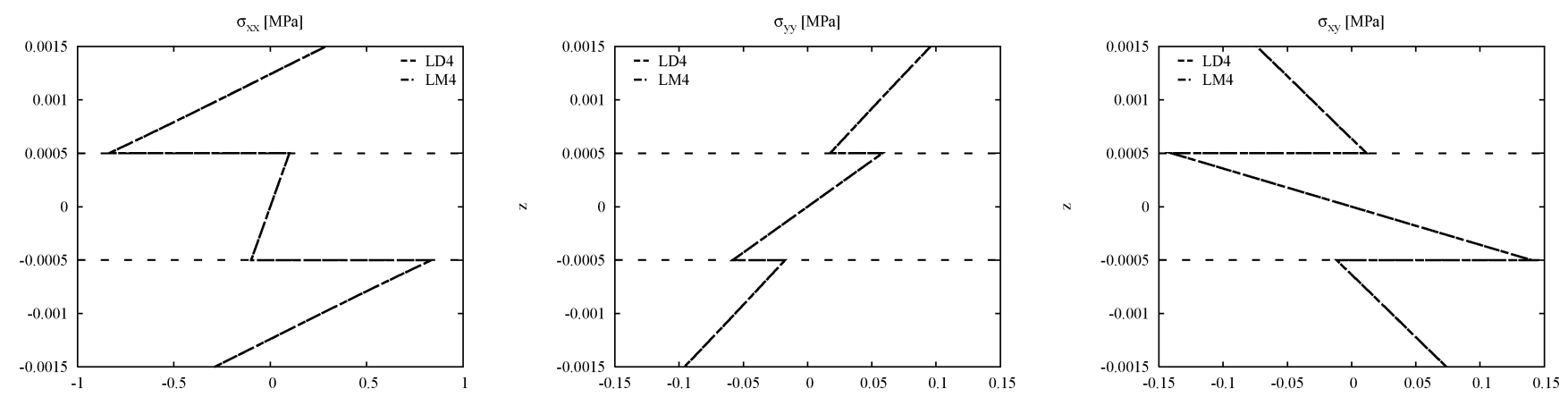

(b) In-plane stress components
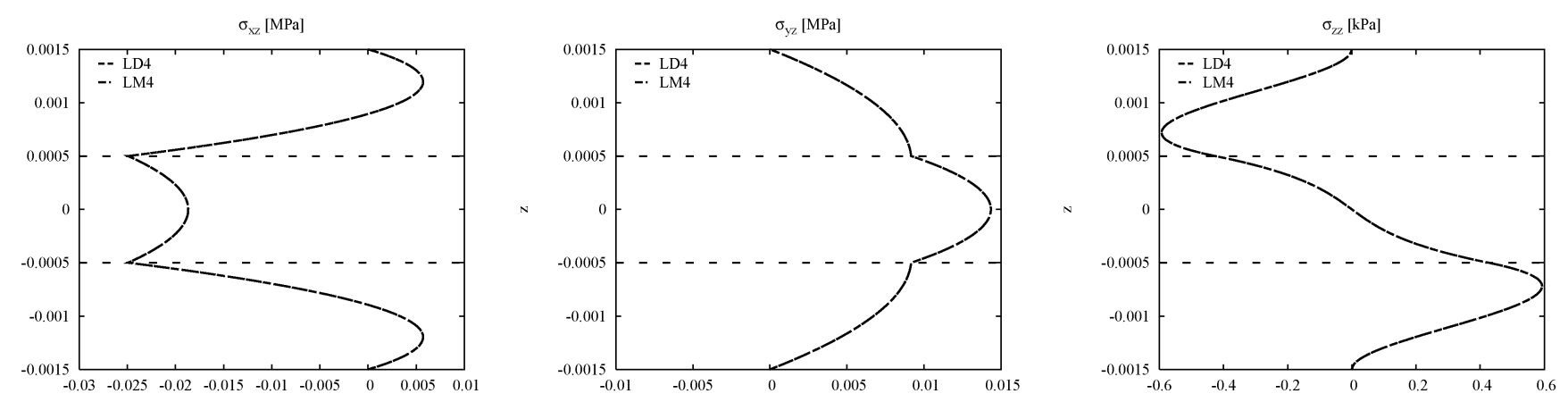

(c) Transverse stress components

FIG. 4. Results of the static analysis with the actuator configuration: mechanical response.

$\delta \hat{\Phi}_{\tau}^{k}: \quad \hat{\mathbf{K}}_{v u}^{k \tau s} \hat{\mathbf{u}}_{s}^{k}+\hat{\mathbf{K}}_{v v}^{k \tau s} \hat{\Phi}_{s}^{k}+\hat{\mathbf{K}}_{v s}^{k \tau s}\left(\hat{\boldsymbol{\sigma}}_{n}^{k}\right)_{s}+\hat{\mathbf{K}}_{v d}^{k \tau s}\left(\hat{\mathrm{D}}_{n}^{k}\right)_{s}=\hat{\mathbf{q}}_{e \tau}^{k}$

(20b)

$\delta\left(\hat{\boldsymbol{\sigma}}_{n}^{k}\right)_{\tau}: \quad \hat{\mathbf{K}}_{s u}^{k \tau s} \hat{\mathbf{u}}_{s}^{k}+\hat{\mathbf{K}}_{s v}^{k \tau s} \hat{\Phi}_{s}^{k}+\hat{\mathbf{K}}_{s s}^{k \tau s}\left(\hat{\boldsymbol{\sigma}}_{n}^{k}\right)_{s}+\hat{\mathbf{K}}_{s d}^{k \tau s}\left(\hat{\mathrm{D}}_{n}^{k}\right)_{s}=0$

$\delta\left(\hat{\mathrm{D}}_{n}^{k}\right)_{\tau}: \quad \hat{\mathbf{K}}_{d u}^{k \tau s} \hat{\mathbf{u}}_{s}^{k}+\hat{\mathbf{K}}_{d v}^{k \tau s} \hat{\Phi}_{s}^{k}+\hat{\mathbf{K}}_{d s}^{k \tau s}\left(\hat{\boldsymbol{\sigma}}_{n}^{k}\right)_{s}+\hat{\mathbf{K}}_{d d}^{k \tau s}\left(\hat{\mathrm{D}}_{n}^{k}\right)_{s}=0$

For classical models with only the generalized displacements as independent unknowns, only the first two rows and columns pertaining to the variables $\hat{\mathbf{u}}$ and $\hat{\Phi}$ are present:

$$
\delta \hat{\mathbf{u}}_{\tau}^{k}: \quad \hat{\mathbf{K}}_{u u}^{k \tau s} \hat{\mathbf{u}}_{s}^{k}+\hat{\mathbf{K}}_{u v}^{k \tau s} \hat{\Phi}_{s}^{k}=\hat{\mathbf{M}}^{k \tau s} \ddot{\mathbf{u}}_{s}^{k}+\hat{\mathbf{p}}_{\tau}^{k}
$$

$$
\delta \hat{\Phi}_{\tau}^{k}: \quad \hat{\mathbf{K}}_{v u}^{k \tau s} \hat{\mathbf{u}}_{s}^{k}+\hat{\mathbf{K}}_{v v}^{k \tau s} \hat{\Phi}_{s}^{k}=\hat{\mathbf{q}}_{e \tau}^{k}
$$

The governing equations Eqs. (20) and (21) are expressed in terms of the so-called "fundamental nuclei" of the formulation. These arrays are independent from the laminate description type (i.e., ESL or LW) and are formally invariant with respect to the polynomial terms $\tau, s$. The "fundamental nuclei" for the classical and the advanced formulations are explicitly reported in the Appendix. Appropriate program loops cycling over the thickness polynomials $\tau, s=1, \ldots N$ provide the contributions at layer level. The layer contributions are subsequently assembled at multilayered level by cycling over all 

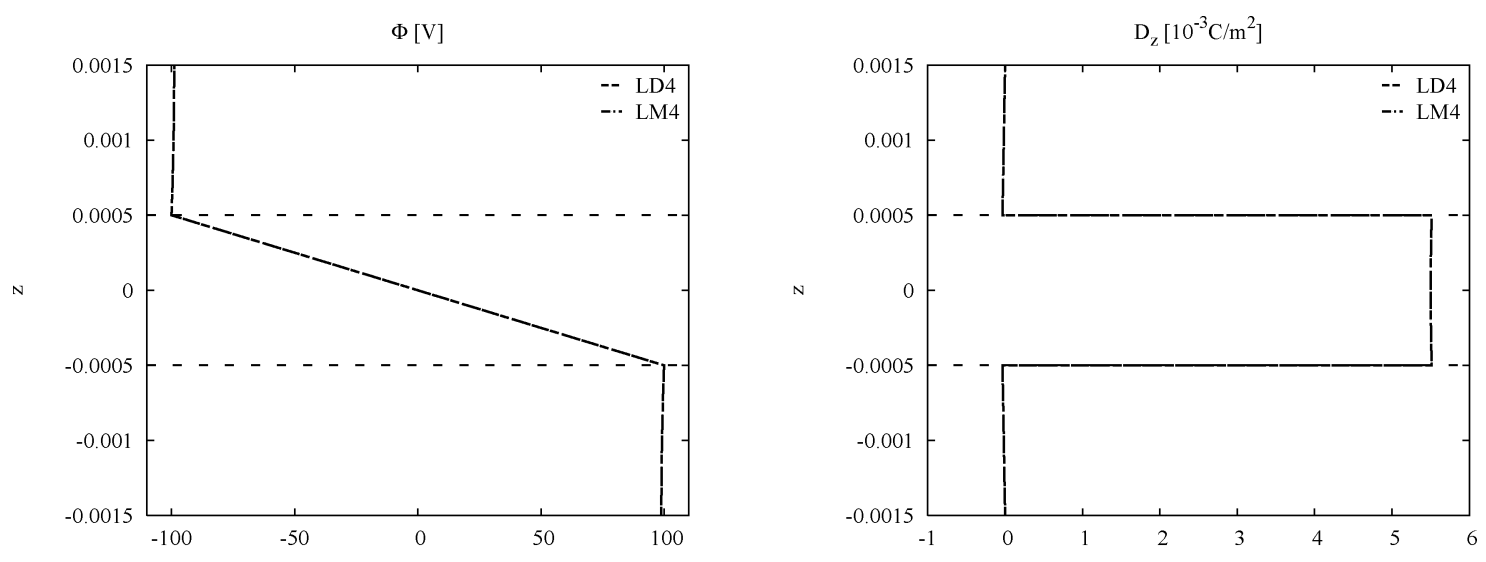

(a) Electric potential and transverse electric displacement
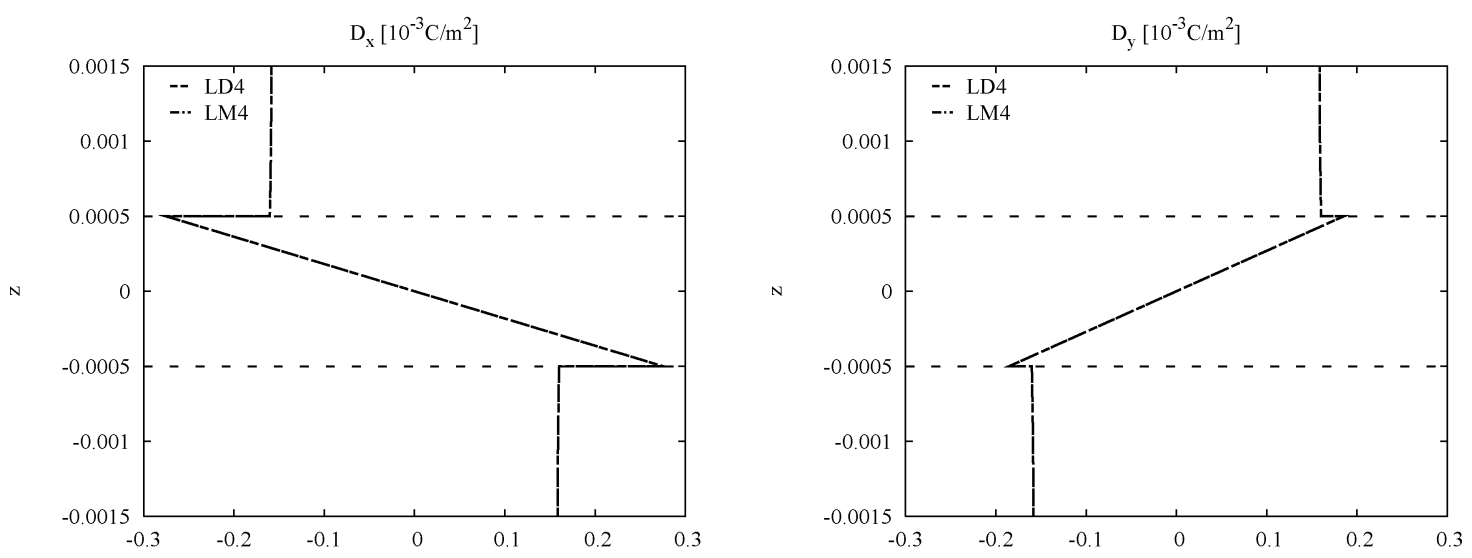

(b) In-plane electric displacement components

FIG. 5. Results of the static analysis with the actuator configuration: electrical response.

layers $k=1, \ldots N_{l}$. The appropriate assembly scheme for each variable depending on the laminate description method must be adopted here. The interlaminar conditions are included according to Section 2.1. at this stage. The loading terms $\hat{\mathbf{p}}_{\tau}^{k}, \hat{\mathbf{q}}_{e \tau}^{k}$ represent the kinematically equivalent distribution of the applied pressure loadings and electric charges, respectively, over the layer DOF. External loadings associated to prescribed displacements or electric potential are applied as Dirichlet conditions on the assembled system. The free-vibration analysis is conducted by solving the purely mechanical eigenvalue problem

$$
\left(\hat{\mathbf{K}}_{m m}-\omega^{2} \hat{\mathbf{M}}\right) \hat{\mathbf{u}}=0
$$

which is obtained after application of all Dirichlet conditions and after all other variables have been statically condensed out. As a consequence, the equivalent "mechanical" stiffness $\hat{\mathbf{K}}_{m m}$ contains all information concerning the electric boundary conditions. This procedure is in conformity to what reported in [54] for the distinction between open-circuited and short-circuited electrode configurations.

\section{NUMERICAL VALIDATION}

The proposed models are compared to exact 3D solutions available in open literature for both static and free-vibration analysis [46, 47]. In all cases, a square three-layered plate of edge length $a=3 \mathrm{~cm}$ and total thickness $h=3 \mathrm{~mm}$ is considered. The piezoceramic PZT-5H layer is placed between two elastic fibre-reinforced layers $\left(0^{\circ}\right.$ Graphite-Epoxy composite, Gr-Ep) and all layers have the same thickness $h_{k}=1 \mathrm{~mm}$. The material data are given in Table 1. Two different configurations are considered in the following (see Figure 3):

- Actuator configuration: a potential difference is prescribed at the top and bottom electrodes of the piezolayer according to the Dirichlet boundary conditions $\Phi^{2 t}=-\bar{\Phi}$ and $\Phi^{2 b}=+\bar{\Phi}$, where $\bar{\Phi}=100 \mathrm{~V}$ (Figure $3(a))$. 

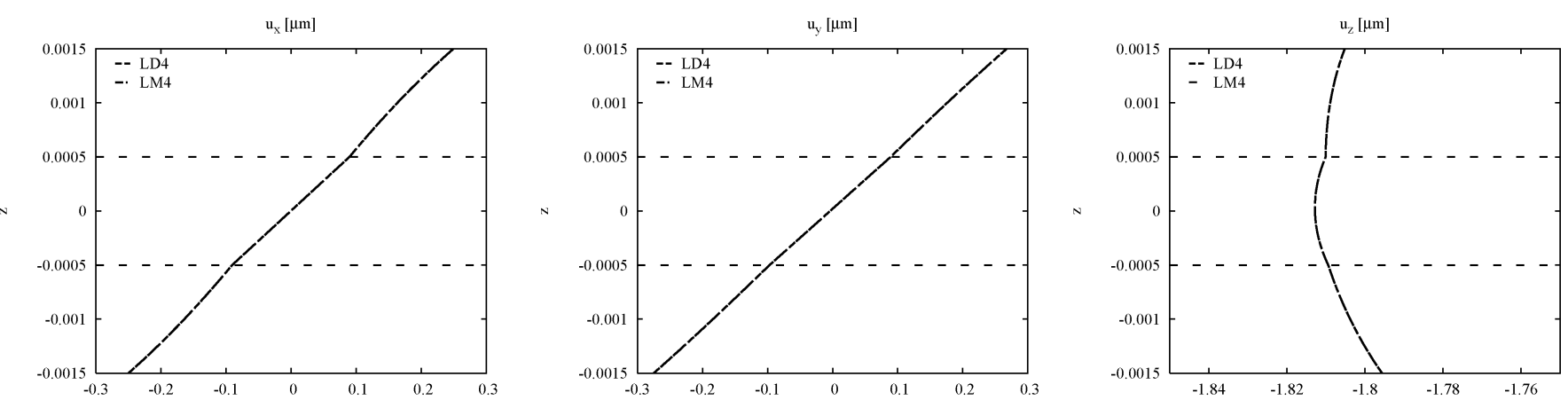

(a) Displacement components
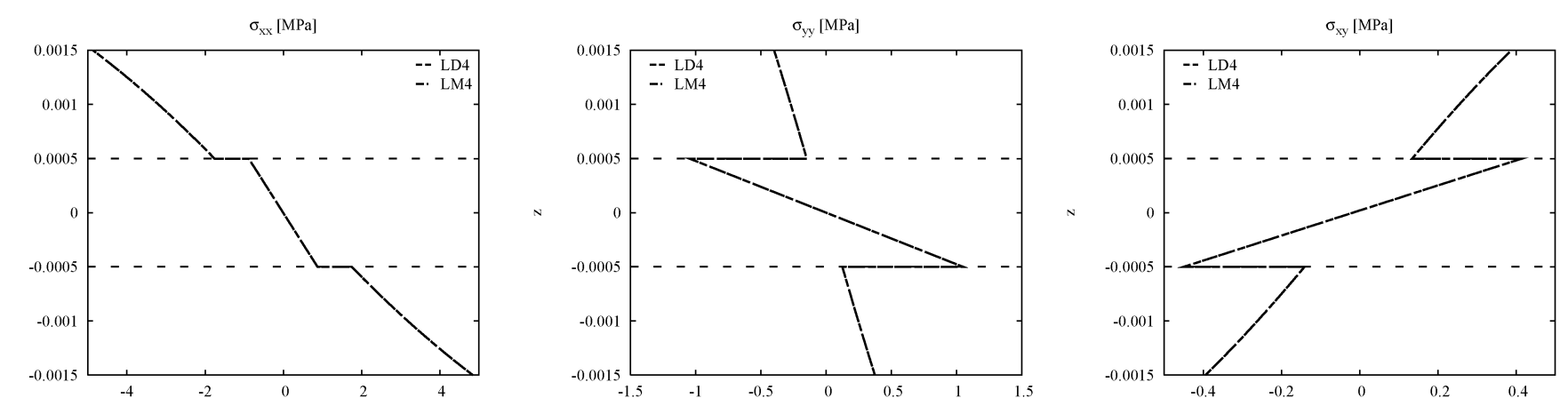

(b) In-plane stress components
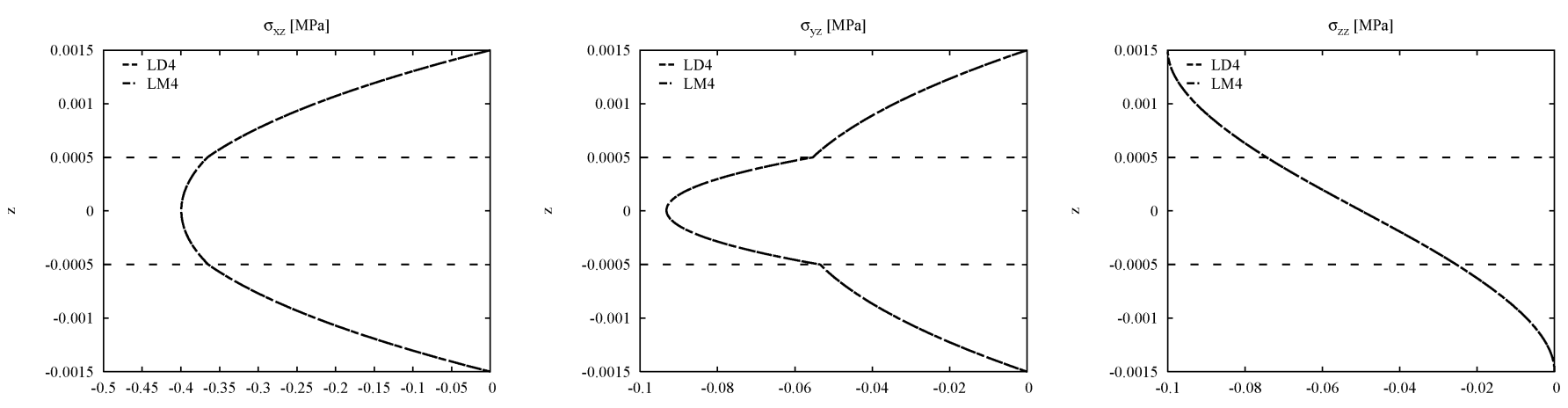

(c) Transverse stress components

FIG. 6. Results of the static analysis with the sensor configuration: mechanical response.

- Sensor configuration: a transverse pressure load defined by Eq. (19a) with $p_{0}=-0.1 \mathrm{MPa}$ is applied on the top surface of the laminate (Figure 3(b)). The electrodes are taken to be in open-circuit condition, i.e., $q=0$ on the top and bottom surfaces of the piezolayers.

Except for the transverse pressure load applied in the sensor configuration, in all cases the top and bottom surfaces of the laminate are stress-free and charge free. For the purpose of validation, we report the results obtained with the most accurate thickness assumptions, which correspond to an LW assumption of 4 th order for all involved variables.

\subsection{Static Analysis}

The results for the actuator and the sensor configurations are depicted in Figures 4-7, respectively. The thickness behaviours of all variables are in excellent agreement with those obtained with the full 3D analysis reported in [46]. In particular, all higher-order, asymmetric thickness variations are correctly captured by the LD4 and LM4 models. Furthermore, all natural boundary conditions (i.e., the vanishing transverse 

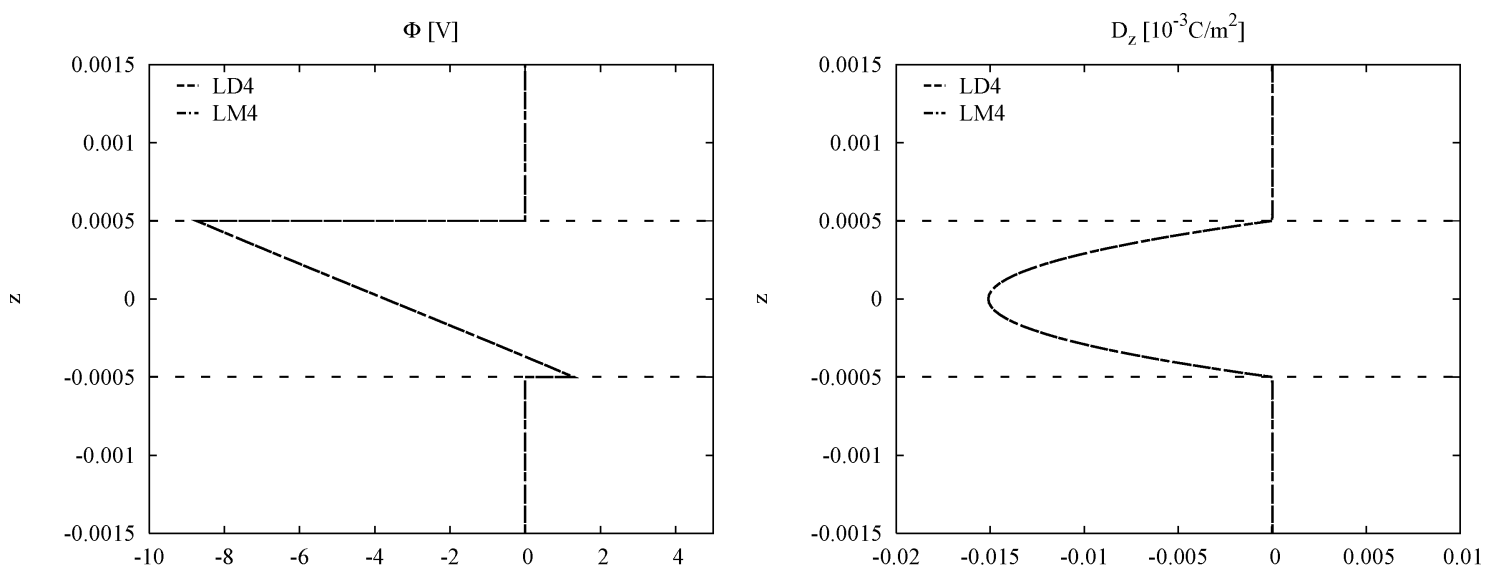

(a) Electric potential and transverse electric displacement
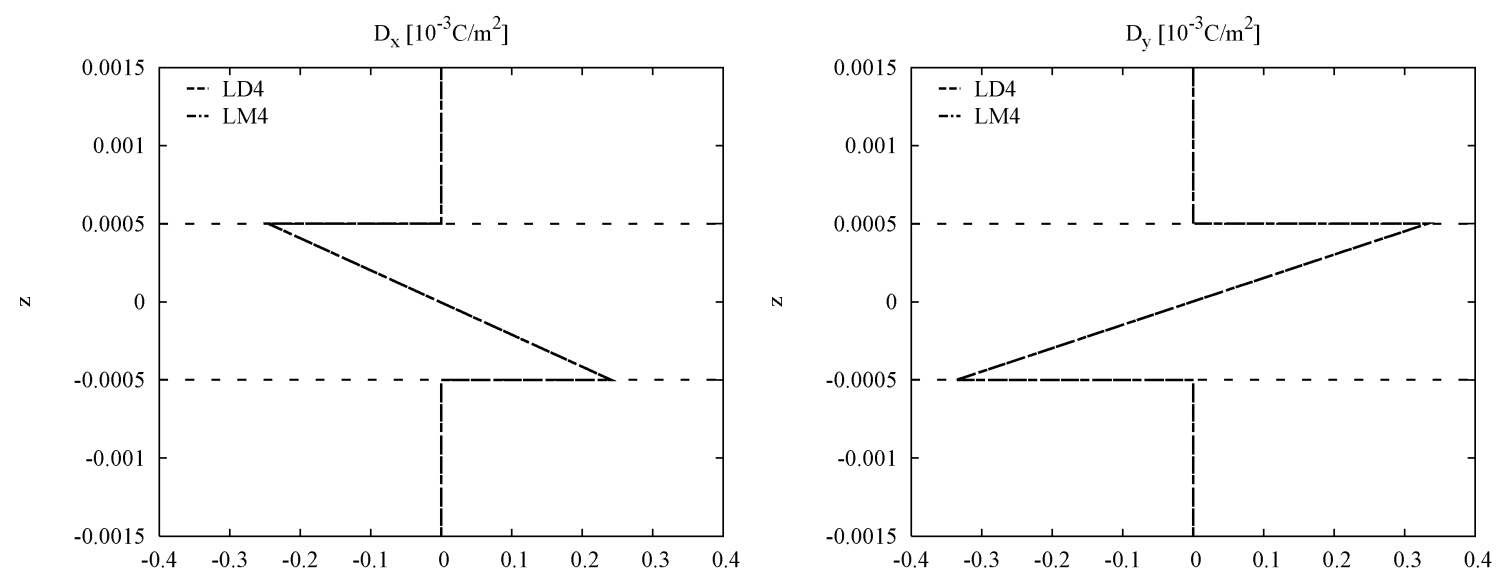

(b) In-plane electric displacement components

FIG. 7. Results of the static analysis with the sensor configuration: electrical response.

shear stresses and transverse electric displacement at the laminate top and bottom as well as the conditions on the transverse normal stress) are accurately reproduced. Spurious oscillations would be obtained if Dirichlet conditions on $\mathrm{D}_{z}$ or $\boldsymbol{\sigma}_{n}$ were imposed. In the present case, the LD4 model is accurate enough for correctly representing the interlaminar conditions discussed in Section 2.1. Hence, the classical and the advanced model are in perfect accordance.

For the sensor configuration in the case study proposed in [46], the interlaminar continuity of the electric displacement comes along with a discontinuity of the electric potential (Figure 7(a)). This behaviour corresponds with an insulated piezoelectric layer and the electric field inside the elastic layers vanishes. However, laminates with embedded piezoceramics do not need an insulation sheet because the insulating capability can be provided by the adjacent elastic layers. For this reason, we complement this case study by a modified sensor configuration with a continuous distribution of the electric potential, in accordance to the definition given in Section 2.1. The comparison between the configuration admitting a "jump" in the electric potential and the modified sensor configuration with continuous $\Phi$ is depicted in Figure 8. Since no difference was recognizable in the mechanical response, only the electric variables have been plotted. It can be seen that the electric coupling between the elastic and the piezoelectric layers due to the electroded interface induces an electric field inside the elastic layers. An asymmetric distribution of the $D_{z}$ is caused by the mechanical loading applied on the top surface of the laminate.

\subsection{Free-Vibration Analysis}

The first five non-dimensional eigenfrequencies $\bar{\omega}=$ $\left(a^{2} / h\right) \sqrt{\left(\rho / C_{22}\right)_{f}}$ (where the subscript $f$ indicates that the mechanical properties are those of the elastic faces) are reported in Table 2 along with the exact 3D solutions reported in [47].

The short-circuit (SC) condition corresponds to the actuator configuration depicted in Figure 3(a) in which $\bar{\Phi}=0 \mathrm{~V}$. The open-circuit (OC) condition corresponds to the sensor 

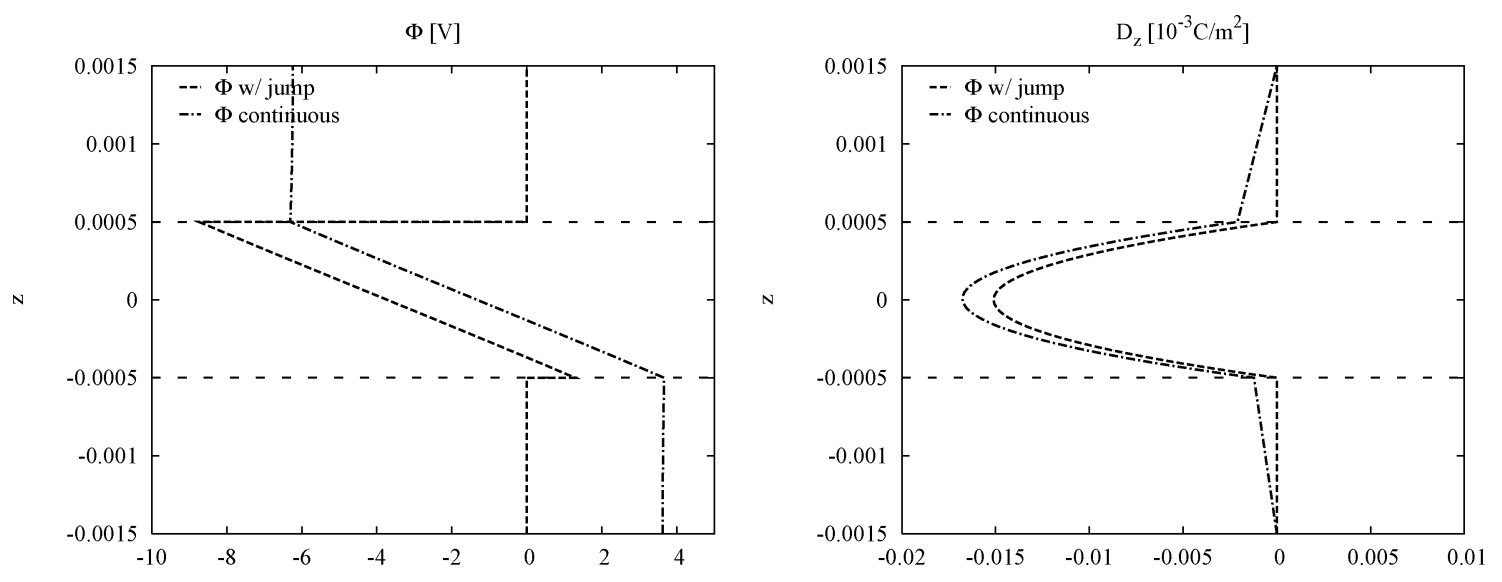

(a) Electric potential and transverse electric displacement
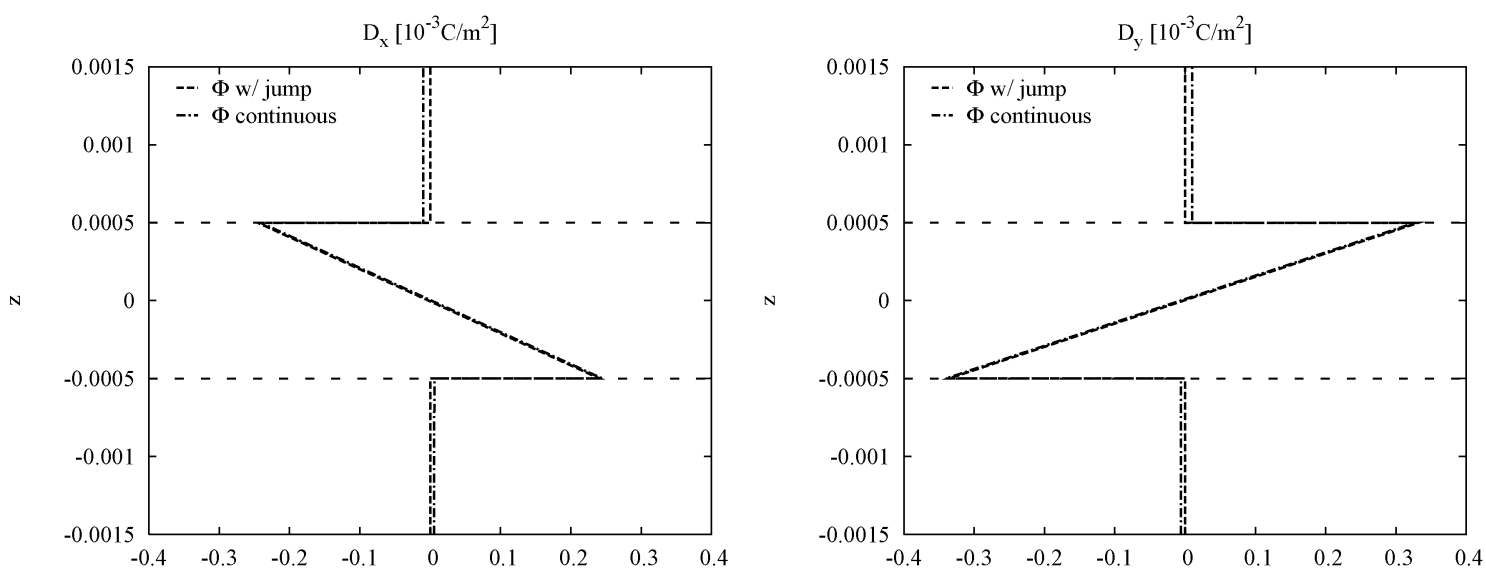

(b) In-plane electric displacement components

FIG. 8. Comparison between the electrical response of the sensor configurations with a continuous and a discontinuous electric potential distribution.

configuration with no mechanical loading (Figure 3(b)). Again, we distinguish the case in which the piezoelectric layer is insulated (i.e., the electric potential can be discontinuous at the electrode) from that in which the electroded interface forces the continuity of the electric potential. While the former configura-

TABLE 2

Non-dimensional frequencies $\bar{\omega}$ and respective EMCC $\kappa$ for the first five vibration modes (first thickness mode: $m_{z}=1$ )

\begin{tabular}{|c|c|c|c|c|c|c|c|}
\hline \multirow{2}{*}{$\begin{array}{l}\text { Mode } \\
\left(m_{x}, m_{y}\right)\end{array}$} & \multicolumn{2}{|c|}{$\begin{array}{c}3 \mathrm{D} \\
{[47]}\end{array}$} & \multicolumn{5}{|c|}{$\begin{array}{c}\text { Advanced } \\
\text { model LM4 }\end{array}$} \\
\hline & SC & $\mathrm{OC}$ & SC & $\mathrm{OC}_{i}$ & $\mathrm{OC}_{c}$ & $\kappa_{i}$ & $\kappa_{c}$ \\
\hline ,1) & 7.834 & 7.893 & 7.834 & 7.893 & 7.893 & 0.122 & 0.122 \\
\hline 1,2$)$ & 11.939 & 11.999 & 11.939 & 11.999 & 11.998 & 0.100 & 0.09 \\
\hline 1,3$)$ & 19.776 & 19.837 & 19.776 & 19.837 & 19.836 & 0.079 & 0.07 \\
\hline$(2,1)$ & 24.030 & 24.588 & 24.030 & 24.588 & 24.580 & 0.212 & 0.21 \\
\hline$(2,2)$ & 26.586 & 27.151 & 26.586 & 27.151 & 27.137 & 0.203 & 0.200 \\
\hline
\end{tabular}

tion is denoted as $\mathrm{OC}_{i}$, the latter is indicated with $\mathrm{OC}_{c}$. Following [55], the electro-mechanical coupling coefficient (EMCC) $\kappa$ can be computed from the open-circuit and short-circuit eigenfrequencies $\omega_{O C}$ and $\omega_{S C}$ as

$$
\kappa=\sqrt{\frac{\omega_{O C}^{2}-\omega_{S C}^{2}}{\omega_{O C}^{2}}}
$$

This is a meaningful parameter for quantifying the electromechanical energy conversion capability and is, hence, a sound indicator of the actuation efficiency of the considered structure. The EMCCs computed on the basis of the $\mathrm{OC}_{i}$ and $\mathrm{OC}_{c}$ configurations are denoted by $\kappa_{i}$ and $\kappa_{c}$, respectively, and are reported in Table 2 also. Since the most accurate classical and advanced models (i.e., LD4 and LM4) provide identical results, only the values obtained from the LM4 model are reported in the table. The perfect agreement of our 2D model with the exact 3D solution of [47] is emphasized. As expected, a higher EMCC 

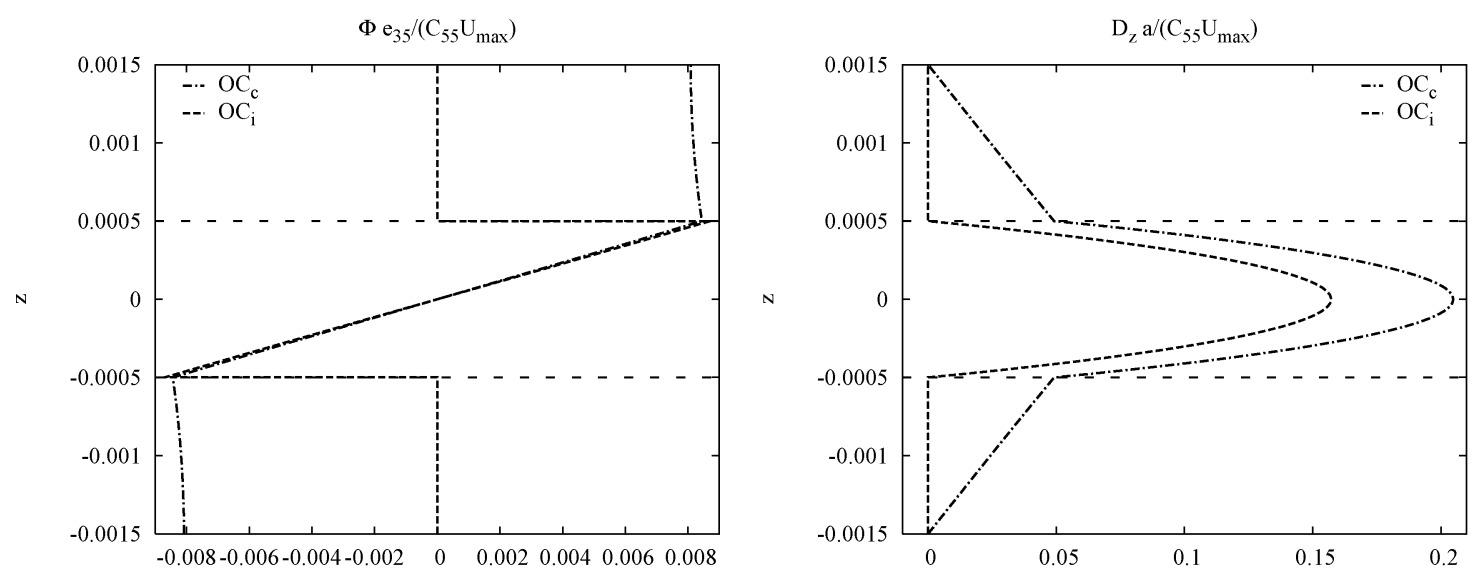

(a) Electric potential and transverse electric displacement
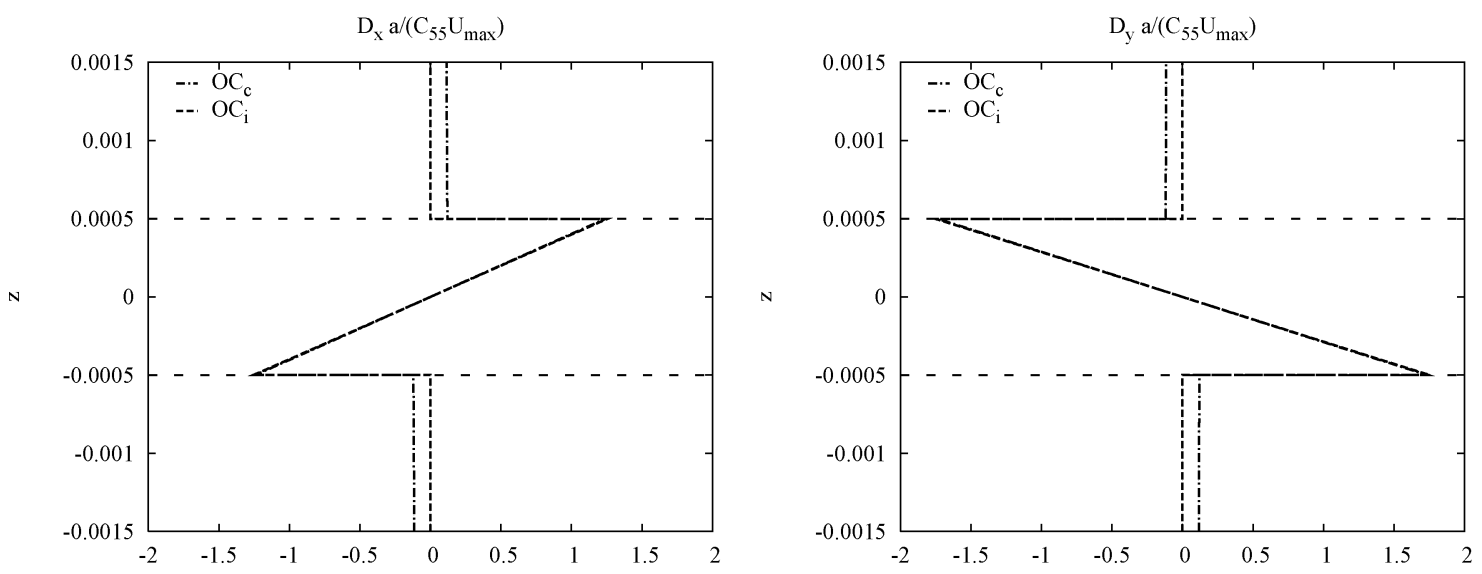

(b) In-plane electric displacement components

FIG. 9. Comparison between the electrical modes associated to the $\mathrm{OC}_{i}$ and the $\mathrm{OC}_{c}$ configurations.

is reached for modes which are characterized by a higher number of waves in the polarization direction ( $x$-axis). Furthermore, the insulated configuration yields in general a higher OC frequency with respect to the $\mathrm{OC}_{c}$ configuration. The comparison between the electrical mode shapes of these two configurations is depicted in Figure 9 (no difference could be recognized in the mechanical modes). The amplitudes have been made nondimensional according to [47]. The existence of an electric field in the elastic layers is easily recognized in the $\mathrm{OC}_{c}$ configuration. On the contrary, this configuration entails a slightly less intense electric field into the piezoelectric layer (Figure 9(a)).

\section{ASSESSMENT OF 2D MODELS}

To demonstrate the capabilities of the Unified Formulation we assess the results obtained with various $2 \mathrm{D}$ models and compare them with an available exact solution. We consider the same free-vibration problem studied in the previous section and for which an exact 3D solution is available [47]. The focus is here put on the first 3 thickness modes of the fundamental in- plane mode $\left(m_{x}=m_{y}=1\right)$. For simplicity, only the shortcircuit configuration is taken into account. Table 3 reports the non-dimensional eigenfrequencies obtained from some selected 2D models. In order to facilitate the assessment, the table contains also the percentage errors

$$
\Delta=100 \frac{\bar{\omega}_{2 D}-\bar{\omega}_{3 D}}{\bar{\omega}_{3 D}} \%
$$

for each mode and for each formulation.

At first, we recall that the exact solution in [47] has shown that the 2nd thickness mode is characterized by the lowest electro-mechanical coupling. This is the reason the errors associated to this mode are in general smaller than those related to Mode 1 and Mode 3. The most accurate 2D models are capable of recovering exactly all 3D solutions. Models with lower-order polynomial approximations or an ESL description of the displacement field show errors of up to 4\%. The results show that, for a given thickness assumption for the displacement field and the electric potential, the advanced models perform better than 
TABLE 3

Non-dimensional frequencies $\bar{\omega}$ and percentage error $\Delta$ for the first three thickness modes $\left(m_{x}=m_{y}=1\right)$ : assessment of various $2 \mathrm{D}$ models

\begin{tabular}{|c|c|c|c|c|c|c|}
\hline & \multicolumn{2}{|c|}{ Mode 1} & \multicolumn{2}{|c|}{ Mode 2} & \multicolumn{2}{|c|}{ Mode 3} \\
\hline & $\bar{\omega}_{1}$ & $\Delta$ & $\bar{\omega}_{2}$ & $\Delta$ & $\bar{\omega}_{3}$ & $\Delta$ \\
\hline 3D (Ref. [47]) & 7.834 & - & 38.355 & - & 76.061 & - \\
\hline LM4 & 7.834 & 0.00 & 38.355 & 0.00 & 76.061 & 0.00 \\
\hline LD4 & 7.834 & 0.00 & 38.355 & 0.00 & 76.061 & 0.00 \\
\hline LM1 & 7.835 & 0.01 & 38.392 & 0.10 & 76.339 & 0.37 \\
\hline LD1 & 8.044 & 2.68 & 38.393 & 0.10 & 76.396 & 0.44 \\
\hline EMZ4 & 7.853 & 0.25 & 38.410 & 0.14 & 76.617 & 0.73 \\
\hline EDZ4 & 7.859 & 0.32 & 38.412 & 0.15 & 76.645 & 0.77 \\
\hline EM4 & 7.942 & 1.38 & 38.428 & 0.19 & 76.410 & 0.46 \\
\hline ED4 & 7.949 & 1.47 & 38.439 & 0.22 & 76.492 & 0.57 \\
\hline EM2 & 7.928 & 1.20 & 38.556 & 0.52 & 76.715 & 0.86 \\
\hline ED2 & 7.962 & 1.63 & 38.573 & 0.57 & 76.822 & 1.00 \\
\hline EMZ1 & 8.022 & 2.40 & 38.447 & 0.24 & 77.367 & 1.72 \\
\hline EDZ1 & 8.132 & 3.80 & 38.448 & 0.24 & 77.368 & 1.72 \\
\hline EM1 & 7.956 & 1.60 & 38.494 & 0.36 & 77.421 & 1.79 \\
\hline ED1 & 8.166 & 4.24 & 38.609 & 0.66 & 77.538 & 1.94 \\
\hline
\end{tabular}

the classical ones. The reason is to be attributed to the refined representation of the piezoelectric interactions due to the independent LW description of the transverse flux variables. Note that a linear approximation of the electric potential inside the piezoelectric layer induces large errors in the classical formulations LD1 and ED1. This error is reduced in the respective partially mixed four-fields formulations LM1 and EM1. The inclusion of the Zig-Zag function improves the accuracy of the result provided the polynomial order is sufficiently high to capture the principal electro-mechanical interactions. This explains the better performance of the EMZ4 model with respect to the EM4 model and the contemporaneous inferior performance of the EMZ1 model in comparison to the EM2 model ${ }^{1}$.

\section{CONCLUSIONS AND OUTLOOK}

In this work we have extended the established Unified Formulation to adaptive structures with shear actuated piezoceramic layers. Classical models based on the piezoelectric Hamilton's principle as well as advanced models derived within a novel partially mixed four-field formulation have been pro-

\footnotetext{
${ }^{1}$ We note that the Zig-Zag function is superimposed only to the displacement field assumption, and that the expansion order $N$ is the same for all unknowns. Therefore, in EMZN and EDZN formulations the electric potential and the transverse fluxes have a polynomial assumption of one order higher than the displacements (e.g., an EDZ1 model has a quadratic assumption for $\Phi$, while the assumption for the displacement field $\mathbf{u}$ consists of a linear polynomial plus the superimposed Zig-Zag function)
}

posed. Advanced models are particularly attractive for the analysis of laminated structures because they ensure a priori the fulfilment of all interlaminar continuity conditions. Since shear actuated piezo-layers are embedded into the adaptive laminate, an appropriate description of the interlaminar conditions is mandatory. Great care has been posed on the definition of physically sound electrical boundary conditions in cases of electroded interfaces. A Navier-type closed-form solution has been presented for the static and free-vibration problems of laminated plates. The validation computations have shown that the most accurate 2D models can precisely recover the exact $3 \mathrm{D}$ solutions. This feature can be exploited by proposing new reference solutions for numerical approximations within, e.g., the Finite Element Method (FEM). An assessment of different thickness assumptions has highlighted the capabilities of the Unified Formulation. The hierarchic order of the implemented models can help to shed light on the requirements posed to an approximate model by complex electro-mechanical interactions. For the presented case study, the advanced formulations have confirmed their superiority with respect to classical ones.

Future studies should promote practically relevant comparisons between piezo-layers actuated in shear and in the more classical extension mode. A FEM implementation of 2D piezoelectric elements based on the Unified Formulation can improve the 3D formulations currently available in commercial software. The hierarchic nature of the Unified Formulation based elements can be conveniently exploited to propose a computationally efficient submodelling technique. These works should make the attractive smart structures technology more ripe to face practical design issues.

\section{REFERENCES}

1. Chopra, I, "Review of state of art of smart structures and integrated systems," AIAA Journal 40, 2145-2187 (2002).

2. Bisegna, P., and Maceri, F., "An exact three-dimensional solution for simply supported R rectangular piezoelectric plates," Journal of Applied Mechanics 63, 628-638 (1996).

3. Gopinathan, S. V., Varadan, V. V., and Varadan, V. K., "A review and critique of theories for piezoelectric laminates," Smart Materials and Structures 9 24-48 (2000).

4. Saravanos, D. A., Heyliger, P. R., "Mechanics and computational models for laminated piezoelectric beams," plates and shells, Applied Mechanics Reviews 52, 305-319 (1999).

5. Benjeddou, A., "Advances in piezoelectric finite element modeling of adaptive structural elements: a survey," Computers \& Structures 76, 347363 (2000)

6. Mitchell, J. A., and Reddy, J. N., "A refined hybrid plate theory for composite laminates with piezoelectric laminae," International Journal of Solids and Structures 32, 2345-2367 (1995).

7. Ossadzow-David, C., and Touratier, M., "A multilayered piezoelectric shell theory," Composites Science and Technology 64, 2121-2137 (2004).

8. Varelis, D., and Saravanos, D. A., "Mechanics and finite elements for nonlinear response of active laminated piezoelectric plates," AIAA Journal $\mathbf{4 2}$ 1227-1235 (2004).

9. Carrera, E., " $C_{z}^{0}$-Requirements-models for the two dimensional analysis of multilayered structures," Composite Structures 37, 373-383 (1997). 
10. Heyliger, P. R., "Traction-free vibration of layered elastic and piezoelectric rectangular parallelepipeds," Journal of the Acoustical Society of America 107, 1235-1245 (2000).

11. Heyliger, P., Ramirez, G., and Saravanos, D. A., "Coupled discrete-layer finite elements for laminated piezoelectric plates," Communications in $\mathrm{Nu}$ merical Methods in Engineering 10, 971-981 (1994).

12. Heyliger, P., Pei, K. C., and Saravanos, D. A., "Layerwise mechanics and finite element model for laminated piezoelectric shells," AIAA Journal 34, 2353-2360 (1996)

13. Carrera, E., "A study of transverse normal stress effect on vibration of multilayered plates and shells," Journal of Sound and Vibration 225, 803 829 (1999).

14. Carrera, E., "Historical review of zig-zag theories for multilayered plates and shells," Applied Mechanics Reviews 56, 287-308 (2003).

15. Reissner, E., "On a certain mixed variational theorem and a proposed application," International Journal for Numerical Methods in Engineering 20, 1366-1368 (1984).

16. Reissner, E., "On a mixed variational theorem and on a shear deformable plate theory," International Journal for Numerical Methods in Engineering 23, 193-198 (1986).

17. Murakami, H., "Laminated composite plate theory with improved in-plane response," Journal of Applied Mechanics 53, 661-666 (1986).

18. Toledano, A., and Murakami, H., "A high-order laminated plate theory with improved in-plane response," International Journal of Solids and Structures 23, 111-131 (1987).

19. Carrera, E., "An assessment of mixed and classical theories on global and local response of multilayered orthotropic plates," Composite Structures 50, 183-198 (2000).

20. Carrera, E., "Developments, ideas and evaluations based upon Reissner's Mixed Variational Theorem in the modeling of multilayered plates and shells," Applied Mechanics Reviews 54, 301-329 (2001).

21. Carrera, E., and Demasi, L., "Classical and advanced multilayered plate elements based upon PVD and RMVT. Part 1: Derivation of finite element matrices. Part 2: Numerical implementations," International Journal for Numerical Methods in Engineering 55, 191-231, 253-291 (2002).

22. Carrera, E., "Theories and finite elements for multilayered plates and shells: A unified compact formulation with numerical assessment and benchmarking," Archives of Computational Methods in Engineering 10, 215-296 (2003).

23. D'Ottavio, M., Wittel, F. K., and Reiser, J., "Mechanism based toolbox: Prototypical implementation of tools," in: Busse, G., B. Kröplin, F. K. Wittel (Eds.), Damage and its Evolution in Fiber-Composite Materials: Simulation and Non-Destructive Evaluation, ISD Verlag, pp. 481-518 (2006).

24. Robbins Jr, D. H., and Reddy, J. N., "Variable kinematic modelling of laminated composite plates," International Journal for Numerical Methods in Engineering 39, 2283-2317 (1996).

25. Robbins Jr, D. H., and Chopra, I., "The effect of laminate kinematic assumptions on the global response of actuated plates," Journal of Intelligent Material Systems and Structures 17, 273-299 (2006).

26. Ballhause, D., D’Ottavio, M., Kröplin, B., and Carrera, E., "A unified formulation to assess multilayered theories for piezoelectric plates," Computers \& Structures 83, 1217-1235 (2005).

27. D'Ottavio, D. Ballhause, B. Kröplin, E. Carrera, "Closed-form solutions for the free-vibration problem of multilayered piezoelectric shells," Computers \& Structures 84, 1506-1518 (2006).

28. Robaldo, A., Carrera, E., and Benjeddou, A., "A unified formulation for finite element analysis of piezoelectric adaptive plates," Computers \& Structures 84, 1494-1505 (2006).

29. Benjeddou, A., "Modelling and simulation of adaptive structures and composites: Current trends and future directions," in: Topping, B., H. V., C. A. Mota-Soares (Eds.), Progress in Computational Structures Technology, Saxe-Coburg Publications, Stirling, UK, pp. 251-280 (2004).

30. D'Ottavio, M., and Kröplin, B., "An extension of Reissner Mixed Variational Theorem to piezoelectric laminates," Mechanics of Advanced Materials and Structures 13, 139-150 (2006).
31. Carrera, E., and Brischetto, S., "Reissner mixed theorem applied to static analysis of piezoelectric shells," Journal of Intelligent Material Systems and Structures 18, 1083-1107 (2007).

32. Carrera, E., and Boscolo, M., "Classical and mixed finite elements for static and dynamics analysis of piezoelectric plates," International Journal for Numerical Methods in Engineering 70, 1135-1181 (2007).

33. Huang, Y.-Q., Liu, M., and Wu, C.-C., "Bimaterial interface hybrid element for piezoelectric laminated analysis," Journal of Strain Analysis 34, 97-106 (1999).

34. Benjeddou, A., and Andrianarison, O., "A piezoelectric mixed variational theorem for smart multilayered composites," Mechanics of Advanced Materials and Structures 12, 1-11 (2005).

35. Carrera, E., and Brischetto, S., "Piezoelectric shells theories with "a priori" continuous transverse electro-mechanical variables," Journal of Mechanics of Materials and Structures 2, 377-399 (2007).

36. Carrera, E., and Fagiano, C., "Mixed piezoelectric plate elements with continuous transverse electric displacement," Journal of Mechanics of Materials and Structures 2, 421-438 (2007).

37. Sun, C.-T., and Zhang, X. D., "Use of thickness-shear mode in adaptive sandwich structures," Smart Materials and Structures 4, 202-206 (1995).

38. Benjeddou, A., Trindade, M. A., and Ohayon, R., 'Piezoelectric actuation mechanisms for intelligent sandwich structures," Smart Materials and Structures 9 328-335 (2000).

39. Zhang, X. D., and Sun, C.-T., "Formulation of an adaptive sandwich beam," Smart Materials and Structures 5, 814-823 (1996).

40. Zhang, X. D., and Sun, C.-T., "Analysis of a sandwich plate containing a piezoelectric core," Smart Materials and Structures 8, 31-40 (1999).

41. Trindade, M. A., and Benjeddou, A., "On higher-order modelling of smart beams with embedded shear-mode piezoceramics actuators and sensors," Mechanics of Advanced Materials and Structures 13, 357-369 (2006).

42. Aldraihem, O. J., and Khdeir, A. A., "Smart beams with extension and thickness-shear piezoelectric actuators," Smart Materials and Structures 9, 1-9 (2000).

43. Vel, S. S., and Batra, R. C., "Exact solution for the cylindrical bending of laminated plates with embedded piezoelectric shear actuators," Smart Materials and Structures 10, 240-251 (2001).

44. Baillargeon, B. P., and Vel, S. S., "Exact solution for the vibration and active damping of composite plates with piezoelectric shear actuators," Journal of Sound and Vibration 282, 781-804 (2005).

45. Vel, S. S., and Baillargeon, B. P., "Analysis of static deformation, vibration and active damping of cylindrical composite shells with piezoelectric shear actuators," Journal of Vibration and Acoustics 127, 395-407 (2005).

46. Benjeddou, A., and Deü, J.-F., "Piezoelectric transverse shear actuation and sensing of plates. Part 1: A three-dimensional mixed state space formulation. Part 2: Application and analysis," Journal of Intelligent Material Systems and Structures 12, 435-449;451-467 (2001).

47. Deü, J.-F., and Benjeddou, A., "Exact free-vibration analysis of laminated plates with embedded piezoelectric transverse shear actuators or sensors," in: Mang, H. A., Rammerstorfer, F. G., J. Eberhardsteiner (Eds.), Proceedings of the Fifth World Congress on Computational Mechanics (WCCM V), Vienna University of Technology, Austria, 2002.

48. American National Standard Institute, IEEE Standard on Piezoelectricity (1987).

49. Tiersten, H. F., Linear Piezoelectric Plate Vibrations, Plenum, New York, 1969.

50. Ikeda, T., Fundamental of Piezoelectricity, Oxford University Press, 1996.

51. Reddy, J. N., Mechanics of Laminated Composite Plates and Shells: Theory and Analysis, 2nd Edition, CRC Press, Boca Raton, FL, 2004.

52. Fernandes, A., and Pouget, J., "An accurate modelling of piezoelectric multi-layer plates," European Journal of Mechanics A/Solids 21, 629-651 (2002).

53. Carrera, E., "On the use of Murakami's zig-zag function in the modeling of layered plates and shells," Computers \& Structures 82, 541-554 (2004).

54. Benjeddou, A., and Belouettar, S., "On the evaluation and application of piezoelectric adaptive structures modal properties," in: Topping, B., H. V., 
Montero, G., R. Montenegro (Eds.), Innovation in Computational Structures Technology, Saxe-Coburg Publications, Ch. 14 (2006).

55. Rogacheva, N. N., The Theory of Piezoelectric Shells and Plates, CRC Press, Boca Raton, 1994.

\section{APPENDIX}

\subsection{Fundamental Nuclei for the Analytical Solution}

The fundamental arrays for both the classical and the advanced formulations are explicitly reported in the following. The layer-specific thickness integrals are first introduced according to

$$
\begin{aligned}
& \left\{E_{\tau s} ; E_{\tau_{z} s} ; E_{\tau s_{z}} ; E_{\tau_{z} s_{z}}\right\} \\
& =\int_{h_{k}}\left\{F_{\tau} F_{s} ; F_{\tau}, F_{s} ; F_{\tau} F_{s}, z ; F_{\tau}, F_{z} F_{s}, z \mathrm{~d} z_{k}\right.
\end{aligned}
$$

Since the arrays relative to the purely mechanical response are formally not affected by the in-plane polarisation scheme, only the piezoelectric arrays are given. ${ }^{2}$ The expression for the purely mechanical arrays (i.e., $\hat{\mathbf{K}}_{u u}^{k \tau s}, \hat{\mathbf{M}}^{k \tau s}, \hat{\mathbf{K}}_{u s}^{k \tau s}, \hat{\mathbf{K}}_{s u}^{k \tau s}$ and $\left.\hat{\mathbf{K}}_{s s}^{k \tau s}\right)$ can be found elsewhere [13, 30, 31].

$$
\begin{aligned}
\hat{\mathbf{K}}_{u v}^{k \tau s} & =\left[\begin{array}{c}
E_{\tau s}\left(m_{x}^{2} e_{11}^{k}+m_{y}^{2} e_{26}^{k}\right)+E_{\tau_{z} s_{z}} k_{35}^{k} \\
E_{\tau s} m_{x} m_{y}\left(e_{12}^{k}+e_{26}^{k}\right) \\
m_{x}\left(-E_{\tau_{z} s} e_{13}^{k}+E_{\tau s_{z}} e_{35}^{k}\right)
\end{array}\right] \hat{\mathbf{K}}_{v u}^{k \tau s} \\
\hat{\mathbf{K}}_{u v}^{k \tau s} & =\left[\hat{\mathbf{K}}_{u v}^{k \tau s}(1,1) \hat{\mathbf{K}}_{u v}^{k \tau s}(1,2) m_{x}\left(-E_{\tau s_{z}} e_{13}^{k}+E_{\tau_{z} s} e_{35}^{k}\right)\right] \\
\hat{\mathbf{K}}_{v v}^{k \tau s} & =\left[-E_{\tau s}\left(m_{x}^{2} \varepsilon_{11}^{s}{ }^{k}+m_{y}^{2} \varepsilon_{22}^{s}{ }^{k}\right)-E_{\tau_{z} s_{z}} \varepsilon_{33}^{s}{ }^{k}\right]
\end{aligned}
$$

The fundamental nuclei of the partially mixed four-field formulation are expressed in terms of the constitutive coefficients defined in Eq. (14) and read

$$
\begin{aligned}
& \hat{\mathbf{K}}_{u v}^{k \tau s}=\left[\hat{\mathbf{K}}_{v u}^{k \tau s}\right]^{T}=\left[\begin{array}{c}
E_{\tau s}\left(m_{x}^{2} e_{p p_{11}}^{M^{k}}+m_{y}^{2} e_{p p_{23}}^{M^{k}}\right) \\
E_{\tau s} m_{x} m_{y}\left(e_{p p_{12}}^{M^{k}}+e_{p p_{23}}^{M^{k}}\right) \\
0
\end{array}\right] ; \\
& \hat{\mathbf{K}}_{u d}^{k \tau s}=\left[\hat{\mathbf{K}}_{d u}^{k \tau s}\right]^{T}=\left[\begin{array}{l}
0 \\
0 \\
0
\end{array}\right] \text {; } \\
& \hat{\mathbf{K}}_{v v}^{k \tau s}=\left[-E_{\tau s}\left(m_{x}^{2} \varepsilon_{p p_{11}}^{M^{k}}+m_{y}^{2} \varepsilon_{p p_{22}}^{M^{k}}\right)\right] ; \quad \hat{\mathbf{K}}_{v d}^{k \tau s}=\left[E_{\tau_{z} s}\right] ; \\
& \hat{\mathbf{K}}_{d v}^{k \tau s}=\left[E_{\tau s_{z}}\right] \\
& \hat{\mathbf{K}}_{s v}^{k \tau s}=\left[\hat{\mathbf{K}}_{v s}^{k \tau s}\right]^{T}=\left[\begin{array}{c}
0 \\
0 \\
-m_{x} E_{\tau s} e_{p n_{13}{ }_{13}}^{k}
\end{array}\right] \\
& \hat{\mathbf{K}}_{s d}^{k \tau s}=\left[\hat{\mathbf{K}}_{d s}^{k \tau s}\right]^{T}=\left[\begin{array}{c}
0 \\
0 \\
-E_{\tau s} e_{n n 12}^{M^{k}}
\end{array}\right] \text {; } \\
& \hat{\mathbf{K}}_{d d}^{k \tau s}=\left[\varepsilon_{n n}^{M^{k}} E_{\tau s}\right] .
\end{aligned}
$$


